\title{
ON SOLUTION SETS OF NONCONVEX DARBOUX PROBLEMS AND APPLICATIONS TO OPTIMAL CONTROL WITH ENDPOINT CONSTRAINTS
}

\author{
H. D. TUAN ${ }^{12}$ \\ (Received 20 September 1993; revised 12 July 1994)
}

\begin{abstract}
We prove a continuous version of a relaxation theorem for the nonconvex Darboux problem $x_{t \tau} \in F\left(t, \tau, x, x_{t}, x_{\tau}\right)$. This result allows us to use Warga's open mapping theorem for deriving necessary conditions in the form of a maximum principle for optimization problems with endpoint constraints. Neither constraint qualification nor regularity assumption is supposed.
\end{abstract}

\section{Introduction}

Consider the optimization problem

$$
f_{0}(x(T, \mathscr{T})) \rightarrow \min
$$

over the solutions of the hyperbolic differential inclusion (or in other words, Darboux problem)

$$
x_{t \tau}(t, \tau) \in F(t, \tau, Z(x)(t, \tau)), \quad(t, \tau) \in \Pi:=[0, T] \times[0, \mathscr{T}],
$$

with boundary conditions

$$
\begin{aligned}
& x(t, 0)=\varphi_{1}(t), \quad t \in[0, T], \\
& x(0, \tau)=\varphi_{2}(\tau), \quad \tau \in[0, \mathscr{T}],
\end{aligned}
$$

and endpoint constraint

$$
f_{1}(x(T, \mathscr{T}))=0 .
$$

'Institute of Mathematics, P.O. Box 631, Bo Ho, Hanoi, Vietnam.

${ }^{2}$ Present address: Dept. of Electronic-Mechanical Eng., Nagoya University, Nagoya 464-01, Japan

(C) Australian Mathematical Society, 1996, Serial-fee code 0334-2700/95 
Here $f_{0}: R^{n} \rightarrow R, F: \Pi \times R^{n} \times R^{n} \times R^{n} \rightarrow 2^{R^{n}}, Z(x)(t, \tau):=\left(x(t, \tau), x_{t}(t, \tau)\right.$, $\left.x_{\tau}(t, \tau)\right)$ and $f_{1}: R^{n} \rightarrow R^{m-1}$.

Note that a constraint of the form $x(T, \mathscr{T}) \in C$, where $C$ is a closed set, is a special case of (4). In fact, by setting $\left.f_{1}(x(T, \mathscr{T}))=\operatorname{dist}(x(T, \mathscr{T}), C)\right)$ then $x(T, \mathscr{T}) \in C$ is made equivalent to $f_{1}(x(T, \mathscr{T}))=0$, that is, (4) holds.

Optimal control problems for inclusion (2) have been extensively studied in the literature (see, for example, $[1,6,12,13,24,25,29,30,32,35-39,46,52,54]$ ) but so far only without the endpoint constraint. Most authors consider the case where $F$ has a parameterized form, so that inclusion (2) becomes a controlled hyperbolic differential equation of the form

$$
x_{t \tau}(t, \tau)=\phi(t, \tau, Z(x)(t, \tau), v), \quad v \in V,
$$

where $v$ is the control function.

When (5) is linear, that is, the function $\phi$ is linear in respect to $Z(x)(t, \tau)$ and $u$, for this class of problems, necessary optimality conditions (in the form of a maximum principle) as well as computational algorithms have been discussed in [13, 23, 29, $37,39,40,46,52,54]$. In this case it is not difficult to show that the solution set of the controlled system (5) is a compact set and consequently, an optimal solution of problem (1), (3), (5) always exists.

When (5) is nonlinear, the first natural question that arises is whether there exists an optimal solution, and that has been studied in $[4,30,35,36]$. These authors show conditions for function $\phi$ such that the solution set of (5) is compact, and the existence of an optimal solution follows immediately from this fact.

Necessary optimality conditions have also been developed in $[6,11,12,23,26,38]$ mainly under the assumption that the objective function $f_{0}$ is linear and that the map $\phi$ is twice continuously differentiable. Then the adjoint equations in the maximum principle involve the derivatives of second order of the map $\phi$. When the function $f_{0}$ is nonlinear, these authors assume that it is twice continuously differentiable also, since then (1), (3), (5) can be reduced to an optimization problem with a linear objective function $[6,11,12,38]$. Indeed, by introducing the additional variable $z(t, \tau)=f_{0}(x(t, \tau))$, then $z_{t \tau}(t, \tau)=\tilde{f}_{0}(Z(x)(t, \tau))$ for some function $\tilde{f}_{0}$ and $(1)$ is equivalent to $z(T, \mathscr{T}) \rightarrow$ minimum.

A necessary optimality condition involving only derivatives of first order of the map $\phi$ for the optimal control problem (1), (3), (5) has been derived by Suryanrayana [38]. In that paper, (5) is reduced to an equivalent one of Dieudonne-Rashevsky form [6] which is a partial differential equation of first order. Clearly this reduction is possible only under the assumptions that the function $\phi$ is continuous with respect to all variables and that the derivatives $\partial f\left(t, \tau, x^{1}, x^{2}, x^{3}\right) / \partial x^{i}, i=1,2,3$ satisfy Lipschitzian conditions with respect to $\left(x^{1}, x^{2}, x^{3}\right)$. Then a Pontryagin-type maximum principle is obtained in [38], while the adjoint equation is more general than those of 
Cesari [6] and Egorov $[11,12]$ because it does not involve the derivatives of second order of the map $\phi$.

In a previous paper [43], we have derived a Pontryagin-type maximum principle for (1)-(3) in the case when $F$ has convex values. When (2) has the parameterized form (5), it has been shown in [43] that this convexity assumption can be removed and we obtain maximum conditions under much weaker assumptions than those of Suryanryana. Namely, in (1)-(3), we assumed that the function $\phi$ is only measurable in $(t, \tau)$, differentiable (not necessary continuously differentiable) in $\left(x^{1}, x^{2}, x^{3}\right)$, continuous in $v$ and that the objective function $f_{0}$ is only differentiable. Of course, one can show that under Suryanryana's smoothness assumptions, our adjoint equations and Suryanaryana's equations are identical [43].

The optimization problem (1)-(3), where $F$ does not depend on $(t, \tau)$, has also been studied by Mahmudov in [24]. Using the discrete approach of Pchenichnyi [28], this author obtains a Egorov-type necessary condition under the assumption that $F$ has convex values. As in [28] for differential inclusion problems, the convexity assumption is crucial for the convergence of approximated solutions to an optimal one. Moreover, the objective function is also assumed to have a convex approximation in sense of [28] at an optimal solution. It is well known from nonsmooth analysis [8] that Lipschitz nonsmooth functions may not satisfy that assumption.

From general optimal control theory, it is well known that optimization problems with an endpoint constraint like (4) are much harder than those without it. As in nonlinear mathematical programming, many results are often obtained under some kind of constraint qualification (CQ) or regularity assumptions (RA). For example, in optimization problems defined by ordinary differential equations, one has to assume Clarke's calmness condition [8] on the endpoint constraint or Frankowska's surjectivity condition involving a linearized inclusion [14]. These conditions ensure that a reference solution is not isolated, that is, in every neighborhood there is at least one solution of the equation that also satisfies the end constraint. Obviously, because of the very complicated structure of the solution set in an optimal control problem, it is not clear how to check CQ or RA. An approach to these problems without CQ and RA was proposed by Warga in [48, 49] and developed by him [50,51] and other authors $[21,18,22,44,45,56]$. In this spirit, in [44] we also investigated (1)-(4) when $F$ has convex values and the functions $f_{0}, f_{1}$ are continuously differentiable. First, by using a special open-mapping theorem for smooth functions and a continuous approximation technique for convex hyperbolic inclusion [42], we derived a sufficient condition for a given solution $\bar{x}(t, \tau)$ to satisfy

$$
f(\bar{x}(T, \mathscr{T})) \in \operatorname{int} f\left(R_{F}(T, \mathscr{T})\right), \quad f:=\left(f_{0}, f_{1}\right),
$$

where $R_{F}(T, \mathscr{T})$ is the reachable set at point $(T, \mathscr{T})$ of inclusion (2). This condition is $0 \in \operatorname{int} \nabla f(\bar{x}(T, \mathscr{T})) R^{L}(T, \mathscr{T})$, where $R^{L}(T, \mathscr{T})$ is the reachable set of a linearized 
inclusion of inclusion (2) at (T, T). Hence, if $\bar{x}(t, \tau)$ is an optimal solution of (1)-(4), then $f(x(T, \mathscr{T})) \in \partial f\left(R_{F}(T, \mathscr{T})\right)$, which implies $0 \notin$ int $\nabla f(x(T, \mathscr{T})) R^{L}(T, \mathscr{T})$. Futhermore, applying a separation theorem (see, for example, [31, consequence 11.7.3]) yields the existence of $\theta \in R^{m},\|\theta\|=1$ such that

$$
\nabla f(x(T, \mathscr{T}))^{*} \theta \in R^{L}(T, \mathscr{T})^{+} .
$$

Since $F$ has convex values, with a suitably chosen linearized inclusion [43], every vector of cone $R^{L}(T, \mathscr{T})^{+}$corresponds to a solution of adjoint inclusions satisfying the maximum condition. A Pontryagin-type maximum principle for (2), (3), (4) was obtained in [44] as a consequence of (7).

As in problems with ordinary differential equations $[49,22,45]$, in order to be able to apply an open-mapping theorem to derive sufficient conditions for (6), one has to use a continuous approximation of solutions of inclusion (2) by solutions of a linearized inclusion. If $F$ has convex values as in $[44,42]$ the continuous approximation is obtained automatically from a successive approximation process [42, Remark 3.1 and Theorem 4.1]. Moreover, as mentioned above, the convexity of the values of $F$ is also crucial to ensure the existence of a linearized inclusion such that every vector of the positive polar of its reachable set corresponds to a solution satisfying the maximum condition.

As it is obvious from the former results, the following important questions related to (1)-(4) are unanswered so far.

1. What is a maximum principle for (1)-(3) (or (1), (3), (5)) with end constraint (4) when $f_{0}$ and $f_{1}$ are nonsmooth functions?

2. Is the convexity assumption for the values of the map $F$ really needed for deriving a maximum principle for (1)-(4)?

The purpose in the present paper is to answer the two questions above, that is, we investigate (1)-(4), assuming only that $F$ has compact (not necesssary convex) values, while $f_{0}, f_{1}$ are Lipschitzian (not necessary continuously differentiable) functions. Here it worth noting that because of the nonconvexity of its values, the map $F$ may be nonparameterized and so it cannot be reduced to Dieudone-Rashevski form as in $[6,38]$. Hence, even without the endconstraint (4), problem (1)-(3) also becomes much more complicated than those of $[6,38]$. Since $F$ has compact values only, for obtaining a maximum principle for problem (1), (2), (4), we shall develop a continuous approximation technique of solutions of inclusion (2) by solutions of a linearized inclusion of the convexified inclusion

$$
x_{t \tau}(t, \tau) \in \operatorname{co} F(t, \tau, Z(x)(t, \tau)), \quad(t, \tau) \in \Pi .
$$

The basic result we will obtain is a Pontryagin-type maximum principle for the problem (1), (2), (4) with nonconvex-valued map $F$ and nonsmooth (Lipschitzian) objective 
function $f_{0}$, nonsmooth (Lipschitzian) endpoint constraint function $f_{1}$, without CQ and RA. Actually, we will even derive the maximum principle for (1), (2), (4) with nonfixed boundary conditions of the form

$$
\begin{aligned}
x_{t}(t, 0) & \in F_{1}(t, x(t, 0)), \quad t \in \Pi_{1}:=[0, T], \\
x_{\tau}(0, \tau) & \in F_{2}(\tau, x(0, \tau)), \quad \tau \in \Pi_{2}:=[0, \mathscr{T}], \quad x(0,0) \in F_{0},
\end{aligned}
$$

where $F_{1}: \Pi_{1} \times R^{n} \rightarrow 2^{R^{n}}, F_{2}: \Pi_{2} \times R^{n} \rightarrow 2^{R^{n}}, F_{0} \subset R^{n}$.

Applying this basic result to (5), we first derive a maximum principle for (1), (5), (4) with nonsmooth data and without any convexity or linearity assumptions.

Our continuous approximation technique of solutions of inclusion (2) by solutions of a linearized inclusion of inclusion (8) is based on a continuous version of a relaxation theorem for inclusion (2) to be proved in the paper. Using this theorem and also Warga's open-mapping principle [48], we shall be able to derive that (6) holds if $0 \in \operatorname{int} \lambda R_{A}(T, \mathscr{T}) \forall \lambda \in \partial_{x} f(\bar{x}(T, \mathscr{T}))$, where $\partial_{x} f(\bar{x}(T, \mathscr{T}))$ denotes Clarke's generalized Jacobian of $f$ at $\bar{x}(T, \mathscr{T})$ and $R_{A}(T, \mathscr{T})$ is the reachable set of a linearized inclusion of inclusion (8). So if $\bar{x}(t, \tau)$ is an optimal solution of (1), (9), (4) then $0 \notin \operatorname{int} \lambda R_{A}(T, \mathscr{T})$ for some $\lambda \in \partial_{x} f(\bar{x}(T, \mathscr{T}))$, which yields the existence of $\theta \in R^{m},\|\theta\|=1$ such that $\lambda^{*} \theta \in R_{A}(T, \mathscr{T})^{+}$. But in inclusion (8), $\operatorname{co} F$ already has convex values and so as in [43], a maximum principle for (1)-(4) follows by choosing a suitable linearized inclusion of inclusion (8) such that every vector of $R_{A}(T, \mathscr{T})^{+}$ corresponds to a solution of adjoint inclusions satisfying the maximum condition.

Aside from serving to derive a maximum principle for (1)-(4), the relaxation theorem has an independent interest. Futhermore, it plays an important role in the study of optimization problems involving (5) with relaxed controls, which will be considered in a subsequent paper (see for example [39] for the definition of relaxed controls of (5)). We mention also that even in its simplest form, our relaxation theorem is a nontrivial extension of the Filippov-Wazewski relaxation theorem for differential inclusions. Indeed, for differential inclusions, it is known (see for example $[2,17,19]$ ) that the Filippov-Wazewski relaxation theorem follows from a Filippov theorem and a Lyapunov theorem for Auman integrals. For inclusion (2) we proved also a Filippovtype theorem [44] and when the map $F$ of inclusion (2) does not depend on partial derivatives $x_{t}(t, \tau), x_{\tau}(t, \tau)$, the relaxation theorem for inclusion (2) can also be proved simply by using Lyapunov theorem [41]. However, when $F$ depends on partial derivatives $x_{t}(t, \tau), x_{\tau}(t, \tau)$, it is not clear how we could derive the relaxation theorem because, as will be seen later, in that case the traditional Lyapunov theorem is no longer suitable. In order to obtain a relaxation theorem for inclusion (2) for the latter case, we shall prove a stronger version of the Lyapunov theorem for Auman integrals in the plane (Theorem 3.1).

The organization of the paper is as follows. Section 2 is the problem statement. In Section 3 we prove a Lyapunov theorem for integrals in the plane and some auxiliary 
results. These results are used for the proof of a relaxation theorem stated in Section 4. Section 5 is devoted to the maximum principle for (1), (2), (4), (9) and (1), (4), (5). Finally, in the Appendix, we prove a continuous version of a Filippov-type theorem for inclusion (2).

\section{Problem statement}

In what follows we shall derive a necessary condition for an optimal solution of (1), (2), (4), (9) in a form of the maximum principle. The following assumptions are made.

A.1. The map $F$ is $\mathscr{L} \otimes \mathscr{B}\left(R^{3 n}\right)$-measurable with compact values and there is a constant $M$ such that

$\operatorname{haus}\left(O_{n}, F\left(t, \tau, x^{1}, x^{2}, x^{3}\right)\right) \leq M$ for a.e. $(t, \tau) \in \Pi$, for every $x^{1}, x^{2}, x^{3} \in R^{n}$.

A.2. For every $t, \tau$ the map $F(t, \tau, \cdot, \cdot, \cdot)$ is Lipschitzian with Lipschitz constant $k>0$, that is,

$$
\begin{aligned}
& \operatorname{haus}\left(F\left(t, \tau, u^{1}, p^{1}, q^{1}\right), F\left(t, \tau, u^{2}, p^{2}, q^{2}\right)\right) \\
& \quad \leq k\left(\left\|u^{1}-u^{2}\right\|+\left\|p^{1}-p^{2}\right\|+\left\|q^{1}-q^{2}\right\|\right), \quad \forall t, \tau, u^{i}, p^{i}, q^{i}(i=1,2) .
\end{aligned}
$$

A.3. The function $f:=\left(f_{0}, f_{1}\right)$ is Lipschitzian with Lipschitz constant $k$. By $\partial_{x} f(\bar{x}(T, \mathscr{T}))$ denote its Clarke's generalized Jacobian at $\bar{x}(T, \mathscr{T})$.

A.4. For every $u \in R^{n}$ the maps $F_{1}(\cdot, u), F_{2}(\cdot, u)$ are measurable with compact values and there are functions $\tilde{\eta}_{1}, \tilde{\eta}_{2}$ integrable on $\Pi_{1}$ and $\Pi_{2}$ such that

$$
F_{1}(t, 0) \subset \tilde{\eta}_{1}(t) S_{n} \forall t \in \Pi_{1}, \quad F_{2}(\tau, 0) \subset \tilde{\eta}_{2}(\tau) S_{n} \quad \forall \tau \in \Pi_{2} .
$$

A.5. For every $(t, \tau) \in \Pi$, the maps $F_{1}(t, \cdot), F_{2}(\tau, \cdot)$ are Lipschitzian with Lipschitz constant $k$.

Throughout the paper, by a solution to the inclusion (2), (9) we mean an absolutely continuous function $u(t, \tau)$ on $\Pi$ with an integrable derivative $u_{t \tau}(t, \tau)$ satisfying inclusion (2) almost everywhere (a.e.) on $\Pi$ and satisfying the boundary conditions (9) a.e. on $\Pi_{1}$ and $\Pi_{2}$.

We refer the reader to [33] for the definition and the main properties of absolutely continuous functions of two variables. In particular, a function $u$ is absolutely continuous on $\Pi$ if and only if it can be expressed in the form

$$
u(t, \tau)=\int_{0}^{t} \int_{0}^{\tau} f(\bar{t}, \bar{\tau}) d \bar{\tau} d \bar{t}+\int_{0}^{t} g(\bar{t}) d \bar{t}+\int_{0}^{\tau} h(\bar{\tau}) d \bar{\tau}+u(0,0) \forall(t, \tau) \in \cdot \Pi,
$$


with $f(\cdot, \cdot) \in L^{1}(\Pi), g(\cdot) \in L^{1}\left(\Pi_{1}\right), h(\cdot) \in L^{1}\left(\Pi_{2}\right)$. Hence the space $Q$ of absolutely continuous functions $u: \Pi \rightarrow R^{n}$ becomes a Banach space when endowed with the norm

$$
\begin{aligned}
\|u(\cdot, \cdot)\|_{Q}= & \|u(0,0)\|+\int_{0}^{T}\left\|u_{t}(t, 0)\right\| d t \\
& +\int_{0}^{\mathscr{T}}\left\|u_{\tau}(0, \tau)\right\| d \tau+\int_{0}^{T} \int_{0}^{\mathscr{T}}\left\|u_{t \tau}(t, \tau)\right\| d \tau d t .
\end{aligned}
$$

In the sequel, $\operatorname{Comp} R^{n}$ (Conv $R^{n}$ respectively) will denote the collection of all nonempty compact (convex and compact, respectively) subsets of $R^{n}$ equipped with the Hausdorff metric haus $(\cdot, \cdot), K^{+}$the positive polar of a set $K$ in Banach space $X$, that is,

$$
K^{+}=\left\{h \in X^{*}:\langle h, a\rangle \geq 0 \quad \forall a \in K\right\},
$$

and $L^{1}\left(\widetilde{D}, R^{\kappa}\right)\left(L^{\infty}\left(\widetilde{D}, R^{\kappa}\right)\right.$, respectively) the space of integrable (measurable and essentially bounded [55, page 34], respectively) functions from $\widetilde{D}$ to $R^{\kappa}$. When for the sake of simplicity we take $\kappa=n$, we write $L^{1}(\widetilde{D})$ instead of $L^{1}\left(\widetilde{D}, R^{n}\right)$. For any set-valued map $G, \operatorname{co} G$ denotes the map $u \rightarrow \operatorname{co} G(u)$ (closed convex hull of $G(u)$ ). Also the following notation will be used:

$W_{1}$ and $W_{2}$ : Banach spaces of absolutely continuous functions $\tilde{u}: \Pi_{1} \rightarrow R^{n}, \tilde{u}$ : $\Pi_{2} \rightarrow R^{n}$ endowed with the norms

$$
\|\tilde{u}\|_{W_{1}}=\tilde{u}(0)+\int_{\Pi_{1}}\left\|\frac{d}{d t} \tilde{u}(t)\right\| d t, \quad\|\tilde{u}\|_{W_{2}}=\tilde{u}(0)+\int_{\Pi_{2}}\left\|\frac{d}{d \tau} \tilde{u}(\tau)\right\| d \tau
$$

$Z(u)(t, \tau):=\left(u(t, \tau), u_{t}(t, \tau), u_{\tau}(t, \tau)\right) \forall u \in Q ;$

mes $(D)$ : Lebesgue measure of subset $D$ of $R^{2}$ (or $R^{1}$, depending upon the context); $D_{\mid t}=\{\tau:(t, \tau) \in D\}, D_{\mid \tau}=\{t:(t, \tau) \in D\}$ for every $D \subset \Pi$. Obviously, $\Pi_{\mid t}=\Pi_{2}, \Pi_{1 \tau}=\Pi_{1} \forall(t, \tau) \in \Pi$;

$O_{n}$ : the null element of $R^{n}$ and $S_{n}(D ; a)=\{x, \operatorname{dist}(x, D) \leq a\}$ for $D \subset R^{n}$. If there is no confusion we also write 0 instead of $O_{n}$ and $S_{n}$ instead of $S_{n}\left(O_{n} ; 1\right)$;

$\chi_{D}(\cdot)$ : characteristic function of $D$;

$\mathscr{E}^{m}=\left\{\gamma=\left(\gamma_{1}, \gamma_{2}, \ldots, \gamma_{m+1}\right): \gamma_{i} \geq 0, i=1,2, \ldots, m+1, \sum_{i=1}^{m+1} \gamma_{i} \leq 1\right\}$;

$A^{*}:$ the adjoint map of a closed convex process $A$ defined by

$$
p \in A^{*} q \Leftrightarrow(-p, q) \in(\text { graph } A)^{+} .
$$

Recall that a closed convex process $\boldsymbol{A}$ is a set-valued map, whose graph is a closed convex cone. 


\section{A Lyapunov theorem and auxiliary results}

In this section we state preliminary results that are needed in the proof of the relaxation theorem of the next section.

The following Lemma 3.1 can be proved simply as Lemma 2 [11].

LEMMA 3.1. Let $\mathscr{J}$ be a positive integer, $K$ be a compact set of a metric space and $T_{j}(x), j=1,2, \ldots, \mathscr{J}, x \in K$ be measurable subsets of $\Pi$ satisfying

$$
\begin{aligned}
& T_{j_{1}}(x) \cap T_{j_{2}}(x)=\emptyset \forall j_{1} \neq j_{2}, \quad \cup_{j=1}^{\mathscr{J}} T_{j}(x)=\Pi, \\
0= & \lim _{x \rightarrow x^{\prime}} \operatorname{mes}\left(T_{j}(x) \Delta T_{j}\left(x^{\prime}\right)\right)=\lim _{x \rightarrow x^{\prime}} \operatorname{mes}\left(T_{j}(x)_{\mid t} \Delta T_{j}\left(x^{\prime}\right)_{\mid t}\right) \\
= & \lim _{x \rightarrow x^{\prime}} \operatorname{mes}\left(T_{j}(x)_{\mid \tau} \Delta T_{j}\left(x^{\prime}\right)_{\mid \tau}\right) \quad \forall j=1,2, \ldots, \mathscr{J} .
\end{aligned}
$$

Let $v_{j}(\cdot, \cdot) \in L^{1}(\Pi), j=1,2, \ldots, \mathscr{J}$, and let $g$ be defined by

$$
g(x)(t, \tau)=\sum_{j=1}^{\mathscr{J}} \chi_{T_{j}(x)}(t, \tau) v_{j}(t, \tau) .
$$

Then $g$ is a continuous map from $K$ into $L^{1}(\Pi)$.

Let $D$ be a rectangle in $\Pi$ and let $\sigma=\left\{I^{1}, I^{2}, \ldots, I^{N}\right\}$ be a given finite covering of $D$ consisting of rectangles $l^{i}, i=1,2, \ldots, N$, satisfying $\cup_{v=1}^{N} I^{\nu}=D$ and $I^{\nu_{1}} \cap I^{\nu_{2}}=\emptyset$ for every $\nu_{1} \neq \nu_{2}$. Denote by $\mathscr{S}\left(D, \sigma, R^{\mathscr{I}}\right)$ the set of measurable functions taking constant values of $R^{\mathscr{J}}$ in each $I^{\nu} \in \sigma$.

Let $\left\{\varphi_{i}(x), i=1,2, \ldots, \mathscr{N}\right\}$ be a family of continuous functions from $K$ to $R_{+}$ satisfying $\sum_{i=1}^{\mathscr{N}} \varphi_{i}(x)=1$ and $\tilde{f}^{i}, i=1,2, \ldots, \mathscr{N}$, be functions of $\mathscr{S}\left(\Pi, \sigma, R^{\mathscr{J}}\right)$. Define a function $\tilde{f}: K \rightarrow L^{1}\left(\Pi, R^{\mathscr{J}}\right)$ by setting $\tilde{f}(x)=\sum_{i=1}^{\mathscr{N}} \varphi_{i}(x) \tilde{f}^{i} \forall x \in K$.

LEMMA 3.2 For every continuous partition of unity $\left\{p_{j}(x)\right\}_{j=1}^{\mathscr{g}}$ in the compact set $K$ there exist sets $T_{j}(x) \subset \Pi, j=1,2, \ldots, \mathscr{J}$, satisfying $(10)$ and such that the following relations hold for every $j=1,2, \ldots, \mathscr{J}$, and $x \in K$.

$$
\begin{array}{rlrl}
\int_{T_{j}(x)} \tilde{f}(x)(t, \tau) d \tau d t & =p_{j}(x) \int_{\Pi} \tilde{f}_{j}(x)(t, \tau) d \tau d t, & \\
\int_{T_{j}(x)_{t}} \tilde{f}(x)(t, \tau) d \tau & =p_{j}(x) \int_{\Pi_{2}} \tilde{f}_{j}(x)(t, \tau) d \tau & & \forall t \in \Pi_{1}, \\
\int_{T_{j}(x)_{t \tau}} \tilde{f}(x)(t, \tau) d t & =p_{j}(x) \int_{\Pi_{1}} \tilde{f}_{j}(x)(t, \tau) d t & & \forall \tau \in \Pi_{2} .
\end{array}
$$




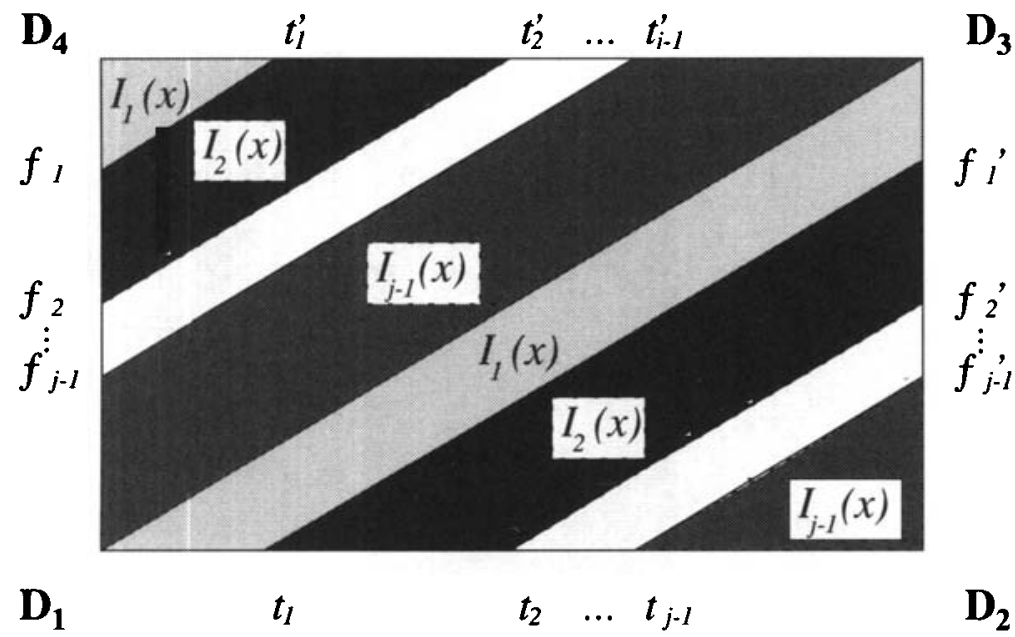

FIGURE 1. The set $I:=D_{1} D_{2} D_{3} D_{4}$ is divided into subsets $I_{1}(x), I_{2}(x), \ldots, I_{\mathscr{g}}(x)$.

PROOF. The proof consists of two steps.

Step 1. Let $I=D_{1} D_{2} D_{3} D_{4}$ be a arbitrary rectangle from $\Pi$. Divide segment $D_{1}{\overline{D_{2}}\left(D_{4}\right.}_{D_{3}}$, respectively) by points $t_{0}, t_{1}, \ldots, t_{\mathscr{g}}\left(t_{0}^{\prime}, t_{1}^{\prime}, \ldots, t_{\mathscr{g}}^{\prime}\right.$, respectively) satisfying $t_{0}=D_{1}\left(t_{0}^{\prime}=D_{4}\right), t_{\mathscr{J}}=D_{2}\left(t_{\mathscr{J}}^{\prime}=D_{3}\right),\left|t_{j}-t_{j-1}\right|=p_{j}(x)\left|D_{1} D_{2}\right|\left(\left|t_{j}^{\prime}-t_{j-1}^{\prime}\right|=\right.$ $\left.p_{j}(x)\left|D_{4} D_{3}\right|\right), j=1,2, \ldots, \mathscr{J}$ (see Figure 1).

We also divide segment $D_{4} D_{1}\left(D_{3} D_{2}\right.$, respectively) by points $\tau_{0}, \tau_{1}, \ldots, \tau_{\mathscr{J}}\left(\tau_{0}^{\prime}, \tau_{1}^{\prime}\right.$, $\ldots, \tau_{\mathscr{J}}^{\prime}$, respectively) satisfying $\tau_{0}=D_{4}\left(\tau_{0}^{\prime}=D_{3}\right), \tau_{\mathscr{J}}=D_{1}\left(\tau_{\mathscr{J}}^{\prime}=D_{2}\right)$, $\mid \tau_{j}$ $\tau_{j-1}\left|=p_{j}(x)\right| D_{4} D_{1} \mid\left(\left|\tau_{j}^{\prime}-\tau_{j-1}^{\prime}\right|=p_{j}(x)\left|D_{3} D_{2}\right|\right)$.

Set

$$
\begin{aligned}
I_{1}(x) & =t_{0} t_{1} \tau_{1}^{\prime} \tau_{0}^{\prime} \cup t_{0}^{\prime} t_{1}^{\prime} \tau_{1} \tau_{0}, \\
& \cdots \\
& \cdots \\
I_{\mathscr{J}}(x) & =t_{\mathscr{J}-1} t_{\mathscr{J}} \tau_{\mathscr{J}}^{\prime} \tau_{\mathscr{J}-1}^{\prime} \cup t_{\mathscr{J}-1}^{\prime} t_{\mathscr{J}}^{\prime} \tau_{\mathscr{J}} \tau_{\mathscr{J}-1},
\end{aligned}
$$

where by $t_{j} t_{j+1} \tau_{j+1}^{\prime} \tau_{j}^{\prime}\left(t_{j}^{\prime} t_{j+1}^{\prime} \tau_{j+1} \tau_{j}\right.$, respectively) we denote the trapezium with vertices $t_{j}, t_{j+1}, \tau_{j+1}^{\prime}, \tau_{j}^{\prime}\left(t_{j}^{\prime}, t_{j+1}^{\prime}, \tau_{j+1}, \tau_{j}\right.$, respectively), $j=0,1,2, \ldots, \mathscr{J}-1$.

It is easy to prove that for every $j=1,2, \ldots, \mathscr{J},(t, \tau) \in I$,

$$
\operatorname{mes}\left(I_{j}\right)=p_{j}(x) \operatorname{mes}(I), \operatorname{mes}\left(I_{j \mid k}\right)=p_{j}(x) \operatorname{mes}\left(I_{\mid \xi}\right), \operatorname{mes}\left(I_{j \mid \tau}\right)=p_{j}(x) \operatorname{mes}\left(I_{\mid \tau}\right) .
$$

We shall prove that $\lim _{x \rightarrow x^{\prime}} \operatorname{mes}\left(I_{j}(x) \Delta I_{j}\left(x^{\prime}\right)\right)=0, j=1,2, \ldots, \mathscr{J}$. Indeed, for every 
$\ell \leq \mathscr{J}$ we have

$$
\lim _{x^{\prime} \rightarrow x} \operatorname{mes}\left(\left(\cup_{j=1}^{\ell} I_{j}(x)\right) \Delta\left(\cup_{j=1}^{\ell} I_{j}\left(x^{\prime}\right)\right)\right)=\lim _{x^{\prime} \rightarrow x}\left|\sum_{j=1}^{\ell}\left(p_{j}(x)-p_{j}\left(x^{\prime}\right)\right)\right| \operatorname{mes}(I)=0,
$$

so

$$
\begin{aligned}
\lim _{x^{\prime} \rightarrow x} \operatorname{mes}\left(I_{j}(x) \Delta I_{j}\left(x^{\prime}\right)\right)= & \lim _{x^{\prime} \rightarrow x} \operatorname{mes}\left(\left(\cup_{j^{\prime}=1}^{j} I_{j^{\prime}}(x) \backslash \cup_{j^{\prime}=1}^{j-1} I_{j^{\prime}}(x)\right) \Delta\right. \\
& \left.\left(\cup_{j^{\prime}=1}^{j} I_{j^{\prime}}\left(x^{\prime}\right) \backslash \cup_{j^{\prime}=1}^{j-1} I_{j^{\prime}}\left(x^{\prime}\right)\right)\right) \\
\leq & \lim _{x^{\prime} \rightarrow x} \operatorname{mes}\left(\left(\cup_{j^{\prime}=1}^{j} I_{j^{\prime}}(x)\right) \Delta\left(\cup_{j^{\prime}=1}^{j} I_{j^{\prime}}\left(x^{\prime}\right)\right)\right) \\
& +\lim _{x^{\prime} \rightarrow x} \operatorname{mes}\left(\left(\cup_{j^{\prime}=1}^{j-1} I_{j^{\prime}}(x)\right) \Delta\left(\cup_{j^{\prime}=1}^{j-1} I_{j^{\prime}}\left(x^{\prime}\right)\right)\right) \\
= & 0 .
\end{aligned}
$$

Analogously, for every $(t, \tau) \in I, j=1,2, \ldots, \mathscr{J}$,

$$
\lim _{x^{\prime} \rightarrow x} \operatorname{mes}\left(I_{j}(x)_{\mid t} \Delta I_{j}\left(x^{\prime}\right)_{\mid l}\right)=0, \quad \lim _{x \rightarrow x^{\prime}} \operatorname{mes}\left(I_{j}(x)_{\mid \tau} \Delta I_{j}\left(x^{\prime}\right)_{\mid \tau}\right)=0 .
$$

Step 2. Let $\sigma=\left\{I^{1}, I^{2}, \ldots, I^{N}\right\}$ be a covering of $\Pi$ consisting of rectangles $I^{j}, j=1,2, \ldots, N$ such that $\tilde{f}^{i}(t, \tau)=\tilde{f}^{i \nu} \forall(t, \tau) \in I^{\nu}, i=1,2, \ldots, \mathscr{N}, \nu=$ $1,2, \ldots, N$. Then from the definition of $f$ we have also $f(x)(t, \tau)=\tilde{f}^{\nu}(x)=$ const for $(t, \tau) \in I^{\nu}$. For every $I^{\nu}, x \in K$, as shown in Step 1 , we can construct $I_{j}^{\nu}(x)$ satisfying

$$
\begin{aligned}
\operatorname{mes}\left(I_{j}^{\nu}(x)\right) & =p_{j}(x) \operatorname{mes}\left(I^{\nu}\right), \\
\operatorname{mes}\left(I_{j}^{\nu}(x)_{\mid \imath}\right) & =p_{j}(x) \operatorname{mes}\left(I_{\mid \ell}^{\nu}\right), \\
\operatorname{mes}\left(I_{j}^{\nu}(x)_{\mid \tau}\right) & =p_{j}(x) \operatorname{mes}\left(I_{\mid \tau}^{\nu}\right) .
\end{aligned}
$$

Set $T_{j}(x)=\cup_{\nu=1}^{N} I_{j}^{\nu}(x)$. Then for every $j=1,2, \ldots, \mathscr{J},(t, \tau) \in \Pi$,

$$
\begin{aligned}
\operatorname{mes}\left(T_{j}(x)\right) & =p_{j}(x) \operatorname{mes}(\Pi), \\
\operatorname{mes}\left(T_{j}(x)_{\mid t}\right) & =p_{j}(x) \operatorname{mes}\left(\Pi_{2}\right), \\
\operatorname{mes}\left(T_{j}(x)_{\mid \tau}\right) & =p_{j}(x) \operatorname{mes}\left(\Pi_{1}\right),
\end{aligned}
$$

and

$$
\begin{aligned}
\lim _{x^{\prime} \rightarrow x} \operatorname{mes}\left(T_{j}(x) \Delta T_{j}\left(x^{\prime}\right)\right) & =\lim _{x^{\prime} \rightarrow x} \operatorname{mes}\left(\left(\cup_{\nu=1}^{N} I_{j}^{\nu}(x)\right) \Delta\left(\cup_{\nu=1}^{N} I_{j}^{\nu}\left(x^{\prime}\right)\right)\right) \\
& \leq \lim _{x^{\prime} \rightarrow x} \operatorname{mes}\left(\cup_{\nu=1}^{N}\left(I_{j}^{\nu}(x) \Delta I_{j}^{\nu}\left(x^{\prime}\right)\right)\right) \\
& \leq \lim _{x^{\prime} \rightarrow x} \sum_{\nu=1}^{N} \operatorname{mes}\left(I_{j}^{\nu}(x) \Delta I_{j}^{\nu}\left(x^{\prime}\right)\right) \\
& =0 .
\end{aligned}
$$


Analogously,

$$
\lim _{x^{\prime} \rightarrow x} \operatorname{mes}\left(T_{j}(x)_{\mid \imath} \Delta T_{j}\left(x^{\prime}\right)_{\mid z}\right)=\lim _{x^{\prime} \rightarrow x} \operatorname{mes}\left(T_{j}(x)_{\mid \tau} \Delta T_{j}\left(x^{\prime}\right)_{\mid \tau}\right)=0 .
$$

Thus the sets $I_{j}(x)$ satisfy $(10)$.

Furthermore,

$$
\begin{aligned}
\int_{T_{j}(x)} \tilde{f}(x) d \tau d t & =\sum_{i=1}^{\mathscr{N}} \varphi_{i}(x) \int_{T_{j}(x)} \tilde{f}^{i}(t, \tau) d \tau d t \\
& =\sum_{i=1}^{\mathscr{N}} \varphi_{i}(x) \sum_{\nu=1}^{N} \int_{l_{j}^{\nu}(x)} \tilde{f}^{i}(t, \tau) d \tau d t=\sum_{i=1}^{\mathscr{N}} \varphi_{i}(x) \sum_{\nu=1}^{N} \operatorname{mes}\left(I_{j}^{\nu}(x)\right) \tilde{f}^{i v} \\
& =p_{j}(x) \sum_{\nu=1}^{N} m e s\left(I^{\nu}\right) \sum_{i=1}^{\mathscr{N}} \varphi_{i}(x) \tilde{f}^{i v}=p_{j}(x) \sum_{\nu=1}^{N} \operatorname{mes}\left(I^{\nu}\right) \tilde{f}^{\nu}(x) \\
& =p_{j}(x) \int_{\Pi} \tilde{f}(t, \tau) d \tau d t,
\end{aligned}
$$

and analogously, for $(t, \tau) \in \Pi$,

$$
\begin{aligned}
& \int_{T_{j}(x)_{\mid t}} \tilde{f}(x) d \tau=p_{j}(x) \int_{\Pi_{2}} \tilde{f}(x) d \tau, \\
& \int_{T_{j}(x)_{\mid \tau}} \tilde{f}(x) d t=p_{j}(x) \int_{\Pi_{1}} \tilde{f}(x) d t .
\end{aligned}
$$

This completes the proof of Lemma 3.2.

The following modification of a Lyapunov theorem for integrals in the plane plays a key role for the proof of the relaxation Theorem.

THEOREM 3.1 Let $G: \Pi \rightarrow$ Comp $R^{n}$ be a measurable map satisfying the condition $\operatorname{haus}\left(O_{n}, G(t, \tau)\right) \leq M<+\infty$ for a.e. $(t, \tau) \in \Pi$.

Then for every $\bar{\epsilon}>0$ and for every measurable function $\bar{v}: \Pi \rightarrow R^{n}$ satisfying

$$
\bar{v}(t, \tau) \in \operatorname{co} G(t, \tau) \text { for almost all }(t, \tau) \in \Pi,
$$

there exists a measurable function $v: \Pi \rightarrow R^{n}$ satisfying

$$
v(t, \tau) \in G(t, \tau) \text { for almost all }(t, \tau) \in \Pi,
$$

and such that

$$
\begin{aligned}
& \max _{(t, \tau) \in \Pi}\left\|\int_{0}^{t} \int_{0}^{\tau}(\bar{v}(\bar{t}, \bar{\tau})-v(\bar{t}, \bar{\tau})) d \bar{\tau} d \bar{t}\right\| \\
& \quad+\max _{\tau \in \Pi_{2}}\left\{e s s \sup _{t \in \Pi_{1}}\left\|\int_{0}^{\tau}(\bar{v}(t, \bar{\tau})-v(t, \bar{\tau})) d \bar{\tau}\right\|\right\} \\
& \quad+\max _{t \in \Pi_{1}}\left\{e s s \sup _{\tau \in \Pi_{2}}\left\|\int_{0}^{t}(\bar{v}(\bar{t}, \tau)-v(\bar{t}, \tau)) d \bar{t}\right\|\right\} \leq \bar{\epsilon} .
\end{aligned}
$$


PROOF. Divide $\Pi_{1}\left(\Pi_{2}\right.$, respectively) into $N$ equal subintervals by the points $t_{i}$ ( $\tau_{i}$, respectively), $i=0,1,2, \ldots, N, t_{0}=\tau_{0}=0, t_{N}=T, \tau_{N}=\mathscr{T}$. The natural number $N$ will be chosen later.

Let $h=\max \{T / N, \mathscr{T} / N\}$ and $\Pi_{i \ell}=\left\{(t, \tau): t_{i} \leq t<t_{i+1}, \tau_{\ell} \leq \tau<\tau_{\ell+1}\right\}$, $i, \ell=0,1,2, \ldots, N-1$. From Lusin's Theorem there exist functions $\epsilon_{1}(h), \epsilon_{2}(h), \epsilon_{3}(h)$ and an at most countable system of rectangles $\left\{\Pi_{\eta}\right\} \subset \Pi$ satisfying

$$
\begin{aligned}
\lim _{h \rightarrow 0} \max \left\{\epsilon_{1}(h), \epsilon_{2}(h), \epsilon_{3}(h)\right\} & =0, \\
\operatorname{int} \Pi_{\eta_{1}} \cap \operatorname{int} \Pi_{\eta_{2}} & =\emptyset \quad \forall \eta_{1} \neq \eta_{2}, \\
\operatorname{mes}\left(\cup_{\eta} \Pi_{\eta}\right) & \geq \operatorname{mes}(\Pi)-h, \\
\operatorname{mes}\left(\left(\Pi \backslash \cup_{\eta} \Pi_{\eta}\right)_{\mid t} \cap \Pi_{2}\right) & \leq \epsilon_{3}(h) \text { for almost all } t \in \Pi_{1}, \\
\operatorname{mes}\left(\left(\Pi \backslash \cup_{\eta} \Pi_{\eta}\right)_{\mid \tau} \cap \Pi_{1}\right) & \leq \epsilon_{3}(h) \text { for almost all } \tau \in \Pi_{2},
\end{aligned}
$$

and such that for $\mu=(i, \ell, \eta), \Pi_{\mu}=\Pi_{i \ell} \cap \Pi_{\eta}$, and

$$
\begin{aligned}
v_{\mu}=\operatorname{mes}\left(\Pi_{\mu}\right)^{-1} \int_{\Pi_{\mu}} \bar{v}(t, \tau) d \tau d t, & (t, \tau) \in \Pi_{\mu} \\
v_{\mu}^{1}(t)=\operatorname{mes}\left(\Pi_{\mu \mid t}\right)^{-1} \int_{\Pi_{\mu \mid t}} \bar{v}(t, \tau) d \tau, & (t, \tau) \in \Pi_{\mu}, \\
v_{\mu}^{2}(\tau)=\operatorname{mes}\left(\Pi_{\mu \mid \tau}\right)^{-1} \int_{\Pi_{\mu \mid \tau}} \bar{v}(t, \tau) d t, & (t, \tau) \in \Pi_{\mu},
\end{aligned}
$$

the following relations hold, whenever $(t, \tau),\left(t, \tau^{\prime}\right) \in \Pi_{\mu}$ with $\operatorname{mes}\left(\Pi_{\mu}\right) \neq 0$.

$$
\begin{aligned}
& \operatorname{haus}\left(G(t, \tau), G\left(t^{\prime}, \tau^{\prime}\right)\right) \leq \epsilon_{1}(h), \\
& \left\|v_{\mu}-v_{\mu}^{1}(t)\right\| \leq \epsilon_{2}(h), \quad\left\|v_{\mu}-v_{\mu}^{2}(\tau)\right\| \leq \epsilon_{2}(h) .
\end{aligned}
$$

By Caratheodory's Theorem, for every $(t, \tau) \in \Pi_{\eta}$ there exist $\alpha_{j}(t, \tau) \geq 0$, $\sum_{j=1}^{n+1} \alpha_{j}(t, \tau)=1$ and $u_{j}(t, \tau) \in G(t, \tau)$ such that

$$
\left\|\sum_{j=1}^{n+1} \alpha_{j}(t, \tau) u_{j}(t, \tau)-\bar{v}(t, \tau)\right\| \leq h .
$$

By virtue of (19), this implies that for $\left(t_{\mu}, \tau_{\mu}\right) \in \Pi_{\mu}$, there exists $\tilde{u}_{j}(t, \tau) \in G\left(t_{\mu}, \tau_{\mu}\right)$ satisfying

$$
\left\|\sum_{j=1}^{n+1} \alpha_{j}(t, \tau) \tilde{u}_{j}(t, \tau)-\bar{v}(t, \tau)\right\| \leq h+\epsilon_{1}(h),
$$

that is, $\bar{v}(t, \tau) \in S_{n}\left(\operatorname{coG}\left(t_{\mu}, \tau_{\mu}\right) ; h+\epsilon_{1}(h)\right)$. By [7, page 288] this yields

$$
v_{\mu} \in S_{n}\left(\operatorname{coG}\left(t_{\mu}, \tau_{\mu}\right) ; h+\epsilon_{1}(h)\right) .
$$


Therefore, again by applying Caratheodory's Theorem, we can find $\alpha_{\mu j} \geq 0, \sum_{j=1}^{n+1} \alpha_{\mu j}$ $=1$ and $u_{\mu j} \in G\left(t_{\mu}, \tau_{\mu}\right)$ satisfying

$$
\left\|v_{\mu}-\sum_{j=1}^{n+1} \alpha_{\mu j} u_{\mu j}\right\| \leq \epsilon_{1}(h)+2 h .
$$

Taking account of (20), we obtain also

$$
\begin{array}{ll}
\left\|v_{\mu}^{1}(t)-\sum_{j=1}^{n+1} \alpha_{\mu j} u_{\mu j}\right\| \leq \epsilon_{1}(h)+\epsilon_{2}(h)+2 h \quad \forall t \in \Pi_{\mu \mid \tau}, \\
\left\|v_{\mu}^{2}(\tau)-\sum_{j=1}^{n+1} \alpha_{\mu j} u_{\mu j}\right\| \leq \epsilon_{1}(h)+\epsilon_{2}(h)+2 h \quad \forall \tau \in \Pi_{\mu \mid t} .
\end{array}
$$

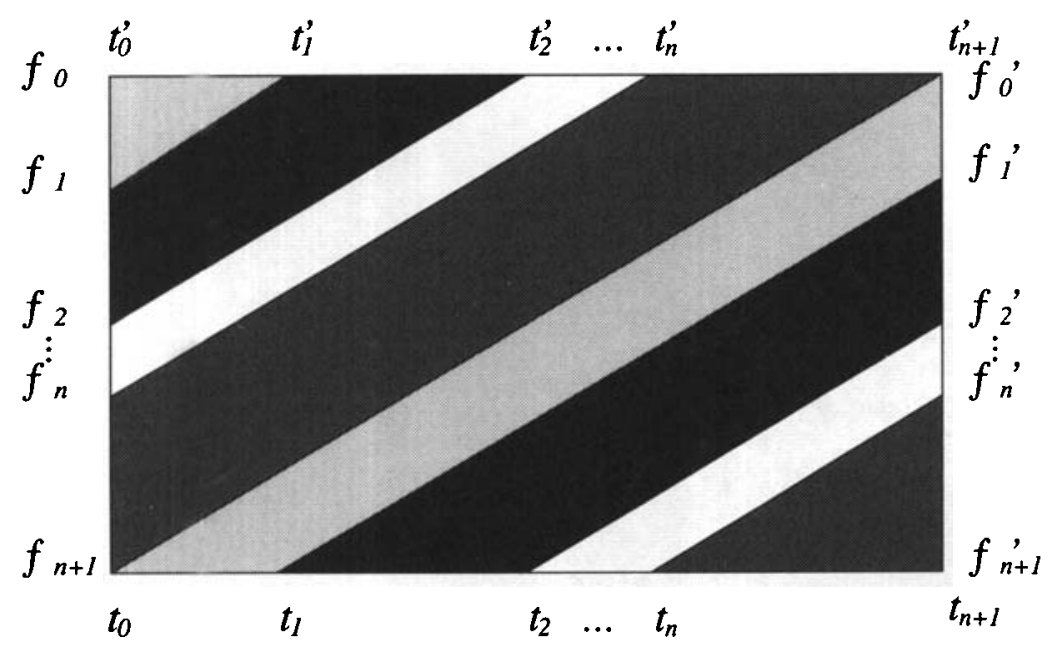

FIGURE 2. The set $\Pi_{\mu}:=t_{0} t_{n+1} t_{n+1}^{\prime} t_{0}^{\prime}$ is divided into subsets $\Pi_{\mu j}, j=1,2, \ldots, n+1$ with $\tau_{n+1}=$ $t_{0}, \tau_{n+1}^{\prime}=t_{n+1}, \quad \tau_{0}^{\prime}=t_{n+1}^{\prime}, \quad \tau_{0}=t_{0}^{\prime}, \frac{t_{j}-t_{j-1}}{t_{n+1}-t_{0}}=\frac{t_{j}^{\prime}-t_{j-1}^{\prime}}{l_{n+1}^{\prime}-t_{0}^{\prime}}=\frac{\tau_{j}-\tau_{j-1}}{\tau_{n+1}-\tau_{0}}=\frac{\tau_{i}^{\prime}-\tau_{j-1}^{\prime}}{\tau_{n+1}^{\prime}-\tau_{0}^{\prime}}=\alpha_{\mu j}, \quad \Pi_{\mu j}=t_{j-1} t_{j} \tau_{j}^{\prime} \tau_{j-1}^{\prime} U$ $t_{j-1}^{\prime} t_{j}^{\prime} \tau_{j} \tau_{j-1}$.

By the method used in Step 1 of the proof of Lemma 3.2 (see Figure 2), for every $\Pi_{\mu}$ we can find a covering $\left\{\Pi_{\mu j}, j=1,2, \ldots, n+1\right\}$ and function $u_{\mu}(t, \tau)$ taking the constant value $u_{\mu j}$ on every $\Pi_{\mu j}$ such that for every $(t, \tau) \in \Pi_{\mu}$,

$$
\sum_{j=1}^{n+1} \alpha_{\mu j} u_{\mu j}=\operatorname{mes}\left(\Pi_{\mu}\right)^{-1} \int_{\Pi_{\mu}} u_{\mu}(t, \tau) d \tau d t
$$




$$
\begin{aligned}
& =\operatorname{mes}\left(\Pi_{\mu \mid t}\right)^{-1} \int_{\Pi_{\mu \mid t}} u_{\mu}(t, \tau) d \tau \\
& =\operatorname{mes}\left(\Pi_{\mu \mid \tau}\right)^{-1} \int_{\Pi_{\mu \mid \tau}} u_{\mu}(t, \tau) d t .
\end{aligned}
$$

Define the measurable function $u_{N}(t, \tau)$ by setting

$$
u_{N}(t, \tau)= \begin{cases}u_{\mu}(t, \tau) & \text { for }(t, \tau) \in \Pi_{\mu} \\ u(t, \tau) & \text { for }(t, \tau) \notin \cup_{\mu} \Pi_{\mu}, \text { where } u(t, \tau) \in G(t, \tau) .\end{cases}
$$

Set $\Delta \bar{v}(t, \tau)=\bar{v}(t, \tau)-u_{N}(t, \tau)$. From (22), (16), (18), (25), for $\ell=1,2, \ldots, N, t \in$ $\Pi_{1}$, we have

$$
\begin{aligned}
\left\|\int_{0}^{\tau_{\ell}} \Delta \bar{v}(t, \tau) d \tau\right\| & \leq 2 M \epsilon_{3}(h)+\sum_{\mu}\left\|\int_{\Pi_{\mu \mid t}}\left(\bar{v}(t, \tau)-u_{N}(t, \tau)\right) d \tau\right\| \\
& =2 M \epsilon_{3}(h)+\sum_{\mu}\left\|\int_{\Pi_{\mu \mid t}}\left(\bar{v}(t, \tau)-u_{\mu}(t, \tau)\right) d \tau\right\| \\
& =2 M \epsilon_{3}(h)+\sum_{\mu}\left\|\operatorname{mes}\left(\Pi_{\mu \mid t}\right) v_{\mu}^{1}(t)-\operatorname{mes}\left(\Pi_{\mu \mid t}\right) \sum_{j=1}^{n+1} \alpha_{\mu j} u_{\mu j}\right\| \\
& \leq \epsilon_{4}(h):=2 M \epsilon_{3}(h)+\mathscr{T}\left(\epsilon_{1}(h)+\epsilon_{2}(h)+2 h\right) .
\end{aligned}
$$

Consequently

$$
\max _{\tau \in \Pi_{2}}\left\{\operatorname{ess} \sup _{t \in \Pi_{1}}\left\|\int_{0}^{\tau} \Delta \bar{v}(t, \bar{\tau}) d \bar{\tau}\right\|\right\} \leq \epsilon_{4}(h)+2 h M \quad \forall(t, \tau) \in \Pi,
$$

and analogously, from (23), (17), (26),

$$
\max _{t \in \Pi_{1}}\left\{\operatorname{ess} \sup _{\tau \in \Pi_{2}}\left\|\int_{0}^{t} \Delta \bar{v}(\bar{t}, \tau) d \bar{t}\right\|\right\} \leq \epsilon_{S}(h)+2 h M
$$

for $\epsilon_{5}(h):=2 M \epsilon_{3}(h)+T\left(\epsilon_{1}(h)+\epsilon_{2}(h)+2 h\right)$, and from (21), (24), (15),

$$
\begin{aligned}
\max _{(l, \tau) \in \Pi}\left\|\int_{0}^{t} \int_{0}^{\tau} \Delta v(\bar{t}, \bar{\tau}) d \bar{\tau} d \bar{t}\right\| & \leq \max _{i, \ell}\left\|\int_{0}^{t_{i}} \int_{0}^{\tau_{\ell}} \Delta v(t, \tau) d \tau d t\right\|+2 h(T+\mathscr{T}) M \\
& \leq \epsilon_{6}(h)+2 h(T+\mathscr{T}) M
\end{aligned}
$$

for $\epsilon_{6}(h):=2 M h+T \mathscr{T}\left(\epsilon_{1}(h)+2 h\right)$.

Now, by choosing the measurable function $v_{h}(t, \tau) \in G(t, \tau)$ for almost every $(t, \tau) \in \Pi$ such that

$$
\begin{aligned}
v_{h}(t, \tau) & =u_{N}(t, \tau) \quad \text { for }(t, \tau) \notin \cup_{\mu} \Pi_{\mu}, \\
\left\|u_{N}(t, \tau)-v_{h}(t, \tau)\right\| & =\operatorname{dist}\left(u_{N}(t, \tau), G(t, \tau)\right) \quad \text { for }(t, \tau) \in \cup_{\mu} \Pi_{\mu},
\end{aligned}
$$


we easily obtain that whenever $(t, \tau) \in \cup_{\mu} \Pi_{\mu}$,

$$
\left\|u_{N}(t, \tau)-v_{h}(t, \tau)\right\| \leq \text { haus }\left(G\left(t_{\mu}, \tau_{\mu}\right), G(t, \mu)\right) \leq \epsilon_{1}(h),
$$

and consequently, for every $(t, \tau) \in \Pi$,

$$
\begin{aligned}
\left\|\int_{0}^{\tau}\left(u_{N}(t, \bar{\tau})-v_{h}(t, \bar{\tau})\right) d \bar{\tau}\right\| & \leq \tau \epsilon_{1}(h), \\
\left\|\int_{0}^{t}\left(u_{N}(\bar{t}, \tau)-v_{h}(\bar{t}, \tau)\right) d \bar{t}\right\| & \leq t \epsilon_{1}(h), \\
\left\|\int_{0}^{t} \int_{0}^{\tau}\left(u_{N}(\bar{t}, \bar{\tau})-v_{h}(\bar{t}, \bar{\tau})\right) d \bar{\tau} d \bar{t}\right\| & \leq t \tau \epsilon_{1}(h) .
\end{aligned}
$$

On the basis of these relations and (27)-(29), we can take a function $\tilde{\epsilon}(h)$ satisfying $\lim _{h \rightarrow 0} \tilde{\epsilon}(h)=0$ and such that

$$
\begin{aligned}
& \max _{(t, \tau) \in \Pi}\left\|\int_{0}^{t} \int_{0}^{\tau}\left(\bar{v}(\bar{t}, \bar{\tau})-v_{h}(\bar{t}, \bar{\tau})\right) d \bar{\tau} d \bar{t}\right\| \\
& \quad+\max _{\tau \in \Pi_{2}}\left\{\operatorname{ess} \sup _{t \in \Pi_{1}}\left\|\int_{0}^{\tau}\left(\bar{v}(t, \bar{\tau})-v_{h}(t, \bar{\tau})\right) d \bar{\tau}\right\|\right\} \\
& \quad+\max _{t \in \Pi_{1}}\left\{\operatorname{ess} \sup _{\tau \in \Pi_{2}}\left\|\int_{0}^{t}\left(\bar{v}(\bar{t}, \tau)-v_{h}(\bar{t}, \tau)\right) d \bar{t}\right\|\right\} \leq \tilde{\epsilon}(h) .
\end{aligned}
$$

Now the statement of the theorem follows by choosing $N$ so large that $\tilde{\epsilon}(h)<\bar{\epsilon}$ and $v(t, \tau)=v_{h}(t, \tau)$. This completes the proof of Theorem 3.1.

\section{A relaxation theorem for hyperbolic inclusions}

Denote by $\mathscr{L}$ the $\sigma$-algebra of the Lebesgue measurable subsets of $\Pi$, and $\mathscr{B}(\Gamma)$ the family of all Borel subsets of a separable Banach space $\Gamma$. Recall that a map $F_{*} \Pi \times \Gamma \rightarrow 2^{R^{n}}$ is called $\mathscr{L} \bigotimes \mathscr{B}(\Gamma)$-measurable if for any closed subset $C$ of $R^{n}$ one has that $\left\{(t, \tau, z) \in \Pi \times \Gamma: F_{*}(t, \tau, z) \cap C \neq \emptyset\right\} \in \mathscr{L} \otimes \mathscr{B}(\Gamma)$.

First, we assume that $G: \Pi \times R^{n} \times R^{n} \times R^{n} \times \Gamma \rightarrow \operatorname{Comp} R^{n}$ and consider the hyperbolic inclusion depending on a parameter

$$
u_{t \tau}(t, \tau) \in G(t, \tau, Z(u)(t, \tau), \gamma)
$$

with the boundary condition

$$
u(t, 0)=u(0, \tau)=0,(t, \tau) \in \Pi .
$$

Suppose that the map $G$ satisfies the following conditions. 
B.1. $G$ is $\mathscr{L} \otimes \mathscr{B}\left(R^{3 n} \times \Gamma\right)$-measurable.

B.2. For every $(t, \tau) \in \Pi, \gamma \in \Gamma$ the map $G(t, \tau, \cdot, \cdot, \cdot, \gamma)$ is Lipschitzian with Lipschitz constant $k$.

B.3. There exists a continuous map $\delta: \Gamma \rightarrow L^{1}(\Pi, R)$ such that haus $\left(O_{n}, G(t, \tau\right.$, $\left.\left.O_{n}, O_{n}, O_{n}, \gamma\right)\right) \leq \delta(\gamma)(t, \tau)$ a.e. in $\Pi$.

B.4. For every $(t, \tau) \in \Pi,\left(x^{1}, x^{2}, x^{3}\right) \in R^{3 n}$ the map $G\left(t, \tau, x^{1}, x^{2}, x^{3}, \cdot\right)$ is continuous.

Now, let $\gamma \rightarrow y(\cdot, \gamma)$ be a given continuous map from $\Gamma$ into $Q$ satisfying

$$
y(t, 0, \gamma)=y(0, \tau, \gamma)=0 \forall t, \tau, \gamma .
$$

Let

$$
Z(y(\gamma))(t, \tau):=\left(y(t, \tau, \gamma), y_{t}(t, \tau, \gamma), y_{\tau}(t, \tau, \gamma)\right)
$$

and

$$
\beta(t, \tau, \gamma)=\operatorname{dist}\left(y_{t \tau}(t, \tau, \gamma), G(t, \tau, Z(y(\gamma))(t, \tau), \gamma)\right)
$$

For the proof of the main result in this section, we need the following theorem, whose proof will be given in the appendix.

THEOREM 4.1 For every $\epsilon>0$, there exists a function $u: \Pi \times \Gamma \rightarrow R^{n}$ such that

(a) for every $\gamma \in \Gamma$ the function $(t, \tau) \rightarrow u(t, \tau, \gamma)$ is a solution of inclusion (30), (31);

(b) the map $\gamma \rightarrow u(\cdot, \cdot, \gamma)$ is continuous from $\Gamma$ into $Q$;

(c) for every $\gamma \in \Gamma,(t, \tau) \in \Pi$,

$$
\begin{aligned}
& \int_{0}^{t} \int_{0}^{\tau}\left\|u_{t \tau}(t, \tau, \gamma)-y_{t \tau}(t, \tau, \gamma)\right\| d \bar{\tau} d \bar{t} \\
& \quad \leq \int_{0}^{t} \int_{0}^{\tau} 2(k+1) \exp (k g(\bar{t}, \bar{\tau}, t, \tau)) \psi_{0}(\gamma)(\bar{t}, \bar{\tau}) d \bar{\tau} d \bar{t}+\epsilon,
\end{aligned}
$$

where

$$
\begin{aligned}
& \psi_{0}(\gamma)(t, \tau)=\operatorname{dist}\left(y_{t \tau}(t, \tau, \gamma), F(t, \tau, Z(y(\gamma))(t, \tau), \gamma),\right. \\
& g(\bar{t}, \bar{\tau}, t, \tau)=6((t-\bar{t})(\tau-\bar{\tau})+(t-\bar{t})+(\tau-\bar{\tau})) .
\end{aligned}
$$

With the choices $\Gamma=Q$ and $y(t, \tau, u)=u(t, \tau)-u(t, 0)-u(0, \tau)+u(0,0) \forall(t, \tau) \in$ $\Pi, u(\cdot, \cdot) \in Q$ and $G\left(t, \tau, x^{1}, x^{2}, x^{3}, u\right)=F\left(t, \tau, x^{1}, x^{2}, x^{3}\right) \forall u \in Q$, Theorem 4.1 yields

COROLlaRY 4.1. For every $\epsilon>0$ there exists a continuous map $r$ from $Q$ to the solution set $\mathscr{R}(F)$ of inclusion (2), (31) such that

$$
\int_{\Pi}\left\|u_{t \tau}(t, \tau)-r(u)_{t \tau}(t, \tau)\right\| d \tau d t \leq \bar{a} \xi(u)+\epsilon \quad \forall u(\cdot, \cdot) \in Q,
$$


where

$$
\begin{gathered}
\bar{a}=2(k+1) \exp (6(T \mathscr{T}+T+\mathscr{T})), \\
\xi(u)=\bar{a} \int_{\Pi}\left[\operatorname { d i s t } \left(u_{t \tau}(t, \tau), G(t, \tau, Z(u)(t, \tau))+k\|u(t, 0)+u(0, \tau)-u(0,0)\|\right.\right. \\
\left.+\left\|u_{t}(t, 0)\right\|+\left\|u_{\tau}(0, \tau)\right\|\right] d \tau d t+\|u(t, 0)+u(0, \tau)-u(0,0)\|
\end{gathered}
$$

Now, we return to inclusion (2), (31) and its convexified inclusion (8), (31). Let $\mathscr{R}(F)$ and $\mathscr{R}(c o F)$ denote the solution sets of inclusions (2), (31) and (8), (31), respectively.

The main result in this section is the following relaxation theorem for inclusion (2), (31).

THEOREM 4.2. Let there be given a compact subset $K \subset \mathscr{R}(c o F) \subset Q$. Then for every $\epsilon>0$ there exists a continuous map $r_{\epsilon}: K \rightarrow \mathscr{R}(F)$ such that $\| r_{\epsilon}(x)(t, \tau)-$ $x(t, \tau) \| \leq \epsilon \forall x \in K,(t, \tau) \in \Pi$.

PROOF. Define

$$
\sigma:=\min \{\epsilon /(4 \bar{a}(10 T \mathscr{T}+7 T+7 \mathscr{T}) T \mathscr{T}), \epsilon / 32\}
$$

with $\bar{a}$ defined from (35) and

$$
\delta=\sigma / M
$$

Divide $\Pi_{1}\left(\Pi_{2}\right)$ into $\mathscr{N}$ equal subintervals by the points $t_{i}\left(\tau_{i}\right.$, respectively) $i=$ $0,1,2, \ldots, \mathscr{N}, t_{0}=\tau_{0}=0, t_{\mathscr{N}}=T, \tau_{\mathscr{N}}=\mathscr{T}$ such that $\left(t_{1}-t_{0}\right)<\delta / T$ and $\left(\tau_{1}-\tau_{0}\right)<\delta / \mathscr{T}$, that is,

$$
\begin{gathered}
\operatorname{mes}\left\{[0, t] \times\left[\tau_{i-1}, \tau_{i}\right]\right\} \leq \delta, \quad \operatorname{mes}\left\{\left[t_{i-1}, t_{i}\right] \times[0, \tau]\right\} \leq \delta \\
\forall(t, \tau) \in \Pi, \quad i=1,2, \ldots, \mathscr{N} .
\end{gathered}
$$

Let $\left\{U_{\sigma / \mathscr{N}^{2}}\left(x^{j}\right)\right\}_{j=1}^{g}$ be a finite covering of $K$ consisting of balls $U_{\sigma / \mathscr{N}^{2}}\left(x^{j}\right)$ of radius $\sigma / \mathscr{N}^{2}$ around $x^{j} \in K$ and let $\{p(x)\}_{j=1}^{\mathscr{J}}$ be its corresponding continuous partition of unity.

By Theorem 3.1 we can choose $v_{j}(t, \tau) \in F\left(t, \tau, Z\left(x^{j}\right)(t, \tau)\right)$ satisfying

$$
\begin{aligned}
& \left\|\int_{t_{i-1}}^{t_{i}} \int_{\tau_{t-1}}^{\tau_{\ell}}\left(x_{t \tau}^{j}(t, \tau)-v_{j}(t, \tau)\right) d \tau d t\right\| \\
& \quad+\text { ess } \sup _{\tau \in\left[\tau_{\ell-1}, \tau_{\ell}\right]}\left\|\int_{t_{i-1}}^{t_{i}}\left(x_{t \tau}^{j}(t, \tau)-v_{j}(t, \tau)\right) d t\right\| \\
& \quad+\text { ess } \sup _{t \in\left[t_{i-1}, t_{i}\right]}\left\|\int_{\tau_{\ell-1}}^{\tau_{\ell}}\left(x_{t \tau}^{j}(t, \tau)-v_{j}(t, \tau)\right) d \tau\right\| \leq \sigma / \mathscr{N}^{2} \mathscr{J} .
\end{aligned}
$$


Take a finite covering $\left\{I^{\nu}\right\}_{v=1}^{N}$ of $\Pi$ consisting of rectangles $I^{\nu}$ and function $\tilde{v}_{j} \in L^{1}(\Pi)$ taking constant values in each $I^{\nu}$ such that

$$
\left\|v_{j}-\tilde{v}_{j}\right\|_{L^{1}(\Pi)} \leq \sigma / \mathscr{N}^{2} \mathscr{J}, j=1,2, \ldots, \mathscr{J} .
$$

Write $I^{\text {vil }}:=I^{\nu} \cap\left(\left[t_{i-1}, t_{i}\right] \times\left[\tau_{\ell-1}, \tau_{\ell}\right]\right)$.

From Lemmas 3.2 and 3.1, for the continuous partition of unity $\{p(x)\}_{j=1}^{\mathscr{J}}$ and for every $I^{\text {vil }}$ there are sets $I_{j}^{\text {vil }} \subset I^{v i \ell}, j=1,2, \ldots, \mathscr{J}$, satisfying the following conditions.

1. $\operatorname{mes}\left(I_{j}^{\text {vil }}(x)\right)=p_{j}(x) \operatorname{mes}\left(I^{\text {vi }}\right)$.

2. For $T_{j}^{i \ell}(x):=\cup_{v=1}^{N} I_{j}^{v i \ell}(x)$,

$$
\begin{aligned}
& \int_{T_{j}^{i^{\ell}(x)}} \tilde{v}_{j}(t, \tau) d \tau d t=p_{j}(x) \int_{t_{i-1}}^{t_{i}} \int_{\tau_{\ell-1}}^{\tau_{\ell}} \tilde{v}_{j}(t, \tau) d \tau d t, \\
& \int_{T_{j}^{i \ell}(x)_{\mid t}} \tilde{v}_{j}(t, \tau) d \tau=p_{j}(x) \int_{\tau_{\ell-1}}^{\tau_{\ell}} \tilde{v}_{j}(t, \tau) d \tau \forall t \in\left[t_{i-1}, t_{i}\right], \\
& \int_{T_{j}^{\ell}(x)_{\mid z}} \tilde{v}_{j}(t, \tau) d t=p_{j}(x) \int_{t_{i-1}}^{t_{i}} \tilde{v}_{j}(t, \tau) d t \forall \tau \in\left[\tau_{\ell-1}, \tau_{\ell}\right] .
\end{aligned}
$$

3. For $T_{j}(x):=\cup_{i=1}^{\mathscr{N}} \cup_{\ell=1}^{\mathscr{N}} T_{j}^{i \ell}(x)$, the map $g: K \rightarrow L^{1}(\Pi)$ defined by $g(x)(t, \tau)=\sum_{j=1}^{g} \chi_{T_{j}(x)}(t, \tau) v_{j}(t, \tau) \forall x \in K,(t, \tau) \in \Pi$ is continuous.

Consider the map $y: K \rightarrow Q$ such that

$$
y(x)(t, \tau)=\int_{0}^{t} \int_{0}^{\tau} g(x)(\bar{t}, \bar{\tau}) d \bar{\tau} d \bar{t} \quad \forall x \in K,(t, \tau) \in \Pi .
$$

From (39-41) for $i=1,2, \ldots, \mathscr{N}, \ell=1,2, \ldots, \mathscr{N}$,

$$
\begin{aligned}
\int_{t_{i-1}}^{t_{i}} \int_{\tau_{t-1}}^{\tau_{\ell}} g(x)(t, \tau) d \tau d t & =\sum_{j=1}^{g} \int_{t_{i-1}}^{t_{i}} \int_{\tau_{\ell-1}}^{\tau_{\ell}} \chi_{T_{j}(x)}(t, \tau) v_{j}(t, \tau) d \tau d t \\
& =\sum_{j=1}^{g} \int_{T_{j}^{\ell}(x)} v_{j}(t, \tau) d \tau d t \\
& \subset \sum_{j=1}^{g} \int_{r_{j}^{i \ell}(x)} \tilde{v}_{j}(t, \tau) d \tau d t+\sigma / \mathscr{N}^{2} . S_{n} \\
& =\sum_{j=1}^{g} p_{j}(x) \int_{t_{i-1}}^{t_{i}} \int_{\tau_{\ell-1}}^{\tau_{\ell}} \tilde{v}_{j}(t, \tau) d \tau d t+\sigma / \mathscr{N}^{2} . S_{n} \\
& =\sum_{j=1}^{g} p_{j}(x) \int_{t_{i-1}}^{t_{i}} \int_{\tau_{\ell-1}}^{\tau_{\ell}}\left(\tilde{v}_{j}(t, \tau)-v_{j}(t, \tau)\right) d \tau d t
\end{aligned}
$$




$$
\begin{array}{r}
+\sum_{j=1}^{\mathscr{J}} p_{j}(x) \int_{t_{i-1}}^{t_{i}} \int_{\tau_{\ell-1}}^{\tau_{\ell}}\left(v_{j}(t, \tau)-x_{t \tau}^{j}(t, \tau)\right) d \tau d t \\
+\sum_{j=1}^{\mathscr{D}} p_{j}(x) \int_{t_{i-1}}^{t_{i}} \int_{\tau_{\ell-1}}^{\tau_{\ell}} x_{t \tau}^{j}(t, \tau) d \tau d t+\sigma / \mathscr{N}^{2} \cdot S_{n} \\
\subset \sum_{j=1}^{\mathscr{J}} p_{j}(x) \int_{t_{i-1}}^{t_{i}} \int_{\tau_{\ell-1}}^{\tau_{\ell}} x_{t \tau}^{j}(t, \tau) d \tau d t+3 \sigma / \mathscr{N}^{2} \cdot S_{n} .
\end{array}
$$

Since

$$
p_{j}(x) \neq 0 \Rightarrow\left\|x-x^{j}\right\|_{Q} \leq \sigma / \mathscr{N}^{2}
$$

we have

$$
\begin{aligned}
& \left\|\int_{t_{i-1}}^{t_{i}} \int_{\tau_{t-1}}^{\tau_{\ell}}\left(g(x)(t, \tau)-x_{t \tau}(t, \tau)\right) d \tau d t\right\| \\
& \quad \leq\left\|\sum_{j=1}^{\mathscr{J}} p_{j}(x) \int_{t_{i-1}}^{t_{i}} \int_{\tau_{\ell-1}}^{\tau_{t}}\left(x_{i \tau}^{j}(t, \tau)-x_{i \tau}(t, \tau)\right) d \tau d t\right\|+3 \sigma / \mathscr{N}^{2} \\
& \quad \leq 4 \sigma / \mathscr{N}^{2} .
\end{aligned}
$$

Analogously, from (39), (42), (43), for $t \in\left(t_{i-1}, t_{i}\right], \tau \in\left(\tau_{\ell-1}, \tau_{\ell}\right]$,

$$
\begin{aligned}
\left\|\int_{\tau_{\ell-1}}^{\tau_{\ell}}\left(g(x)(t, \tau)-x_{t \tau}(t, \tau)\right) d \tau\right\| \leq & \left\|\sum_{j=1}^{J} \int_{T_{j}^{i \ell}(x)_{l}}\left(v_{j}(t, \tau)-\tilde{v}_{j}(t, \tau)\right) d \tau\right\| \\
& +\left\|\sum_{j=1}^{J} p_{j}(x) \int_{\tau_{\ell-1}}^{\tau_{\ell}}\left(\tilde{v}_{j}(t, \tau)-v_{j}(t, \tau)\right) d \tau\right\| \\
& +\left\|\sum_{j=1}^{\mathscr{J}} p_{j}(x) \int_{\tau_{\ell-1}}^{\tau_{\ell}}\left(v_{j}(t, \tau)-x_{t \tau}^{j}(t, \tau)\right) d \tau\right\| \\
& +\left\|\sum_{j=1}^{\mathscr{J}} p_{j}(x) \int_{\tau_{\ell-1}}^{\tau_{\ell}}\left(x_{t \tau}^{j}(t, \tau)-x_{t \tau}(t, \tau)\right) d \tau\right\| \\
\leq & \sigma / \mathscr{N}^{2}+\eta_{1}(t),
\end{aligned}
$$

where, in view of (40), (39) and (44),

$$
\int_{i_{i-1}}^{\ell_{i}} \eta_{1}(t) d t \leq 3 \sigma / \mathscr{N}^{2}, \quad \int_{\tau_{\ell-1}}^{\tau_{t}} \eta_{2}(\tau) d \tau \leq 3 \sigma / \mathscr{N}^{2}
$$


From (45), (37), (38), for $(t, \tau) \in\left(t_{i-1}, t_{i}\right] \times\left(\tau_{\ell-1}, \tau_{\ell}\right]$, we conclude

$$
\begin{aligned}
\|y(x)(t, \tau)-x(t, \tau)\| \leq & \left\|\sum_{i^{\prime}=1}^{i} \sum_{\ell^{\prime}=1}^{\ell} \int_{t_{i^{\prime}-1}}^{t_{i^{\prime}}} \int_{\tau_{\ell^{\prime}-1}}^{\tau_{\ell^{\prime}}}\left(g(x)(t, \tau)-x_{i \tau}(t, \tau)\right) d \tau d t\right\| \\
& +\left\|\int_{0}^{t_{i}} \int_{\tau_{\ell}}^{\tau}\left(g(x)(t, \bar{\tau})-x_{i \bar{\tau}}(t, \bar{\tau})\right) d \bar{\tau} d t\right\| \\
& +\left\|\int_{t_{i}}^{t} \int_{0}^{\tau_{\ell}}\left(g(x)(t, \bar{\tau})-x_{\bar{i} \tau}(\bar{t}, \tau)\right) d \tau d \bar{t}\right\| \\
\leq & \left(4 \sigma / \mathscr{N}^{2}\right) \mathscr{N}^{2}+4 \sigma=8 \sigma .
\end{aligned}
$$

Analogously, from and (46),(47),

$$
\begin{aligned}
\left\|y_{t}(x)(t, \tau)-x_{t}(t, \tau)\right\| \leq & \left\|\sum_{\ell^{\prime}=1}^{\ell} \int_{\tau_{\ell^{\prime}-1}}^{\tau_{\ell^{\prime}}}\left(g(x)(t, \tau)-x_{1 \tau}(t, \tau)\right) d \tau\right\| \\
& +\left\|\int_{\tau_{\ell}}^{\tau}\left(g(x)(t, \bar{\tau})-x_{t \bar{\tau}}(t, \bar{\tau})\right) d \bar{\tau}\right\| \\
\leq & \sigma / \mathscr{N}+\bar{\eta}_{1}(t) \\
\leq & \sigma+\bar{\eta}_{1}(t), \\
\left\|y_{\tau}(x)(t, \tau)-x_{\tau}(t, \tau)\right\| \leq & \sigma / \mathscr{N}+\bar{\eta}_{2}(\tau) \leq \sigma+\bar{\eta}_{2}(\tau),
\end{aligned}
$$

where, in view of (48), (37), (38),

$$
\begin{gathered}
\int_{0}^{t} \bar{\eta}_{1}(\bar{t}) d \bar{t}:=\int_{0}^{t} \eta_{1}(\bar{t}) d \bar{t}+\int_{0}^{t} \int_{\tau_{l}}^{\tau}\left\|g(x)(\bar{t}, \bar{\tau})-x_{\bar{i} \bar{\tau}}(\bar{t}, \bar{\tau})\right\| d \bar{\tau} d \bar{t} \leq 5 \sigma \\
\forall t \in \Pi_{1}, \\
\int_{0}^{\tau} \bar{\eta}_{2}(\bar{\tau}) d \bar{\tau}:=\int_{0}^{\tau} \begin{array}{l}
\left.\eta_{2}(\bar{\tau}) d \bar{\tau}+\int_{t_{i}}^{t} \int_{0}^{\tau} \| g(x)(\bar{t}, \bar{\tau})-x_{\bar{t} \bar{t}}(\bar{t}, \bar{\tau})\right) \| d \bar{\tau} d \bar{t} \leq 5 \sigma \\
\forall \tau \in \Pi_{2} .
\end{array}
\end{gathered}
$$

Now, for every fixed $x \in K,(t, \tau) \in \Pi$, there exists $j \in\{1,2, \ldots, \mathscr{J}\}$ such that $(t, \tau) \in T_{j}(x)$ and $\operatorname{mes}\left(T_{j}(x)\right)>0$. Thus $\left\|x^{j}-x\right\|_{Q} \leq \sigma / \mathscr{N}^{2}$ and furthermore, from (49), (50), (51),

$$
\begin{aligned}
\operatorname{dist}( & g(x)(t, \tau), F(t, \tau, Z(y(x))(t, \tau))) \\
\quad \leq & \operatorname{dist}\left(v_{j}(t, \tau), F\left(t, \tau, Z\left(x^{j}\right)(t, \tau)\right)\right) \\
& +k\left(\left\|Z\left(x-x^{j}\right)(t, \tau)\right\|+\|Z(x-y(x))(t, \tau)\|\right) \\
\leq & k\left(10 \sigma+\bar{\eta}_{1}(t)+\bar{\eta}_{2}(\tau)\right)+k\left\|Z\left(x-x^{j}\right)(t, \tau)\right\| .
\end{aligned}
$$

But it is easily seen that, whenever $\left\|x^{j}-x\right\|_{Q} \leq \sigma / \mathscr{N}^{2} \leq \sigma$,

$$
\int_{\Pi}\left\|Z\left(x-x^{j}\right)(t, \tau)\right\| d \tau d t \leq(T \mathscr{T}+2 T+2 \mathscr{T}) \sigma
$$


Hence, together with (52-54), this implies

$$
\int_{\Pi} \operatorname{dist}(g(x)(t, \tau), F(t, \tau, Z(y(x))(t, \tau))) d \tau d t \leq k \sigma(10 T \mathscr{T}+7 T+7 \mathscr{T}) .
$$

Consequently, by Corollary 4.1 there exists a continuous map $r: Q \rightarrow \mathscr{R}(F)$ such that

$$
\|x(t, \tau)-r(x)(t, \tau)\| \leq \sigma \bar{a}(10 T \mathscr{T}+7 T+7 \mathscr{T}) T \mathscr{T}+\epsilon / 2 \quad \forall x \in y(K) .
$$

Define $r_{\epsilon}: K \rightarrow \mathscr{R}(F)$ by setting $r_{\epsilon}(x)=r(y(x))$. From (55), (49), (36)) we obtain for every $(t, \tau) \in \Pi$ and $x \in K$,

$$
\begin{aligned}
\left\|r_{\epsilon}(x)(t, \tau)-x(t, \tau)\right\| & \leq\|r(y(x))(t, \tau)-y(x)(t, \tau)\|+\|y(x)(t, \tau)-x(t, \tau)\| \\
& \leq \epsilon,
\end{aligned}
$$

completing the proof of Theorem 4.1.

Before closing this section, let us state the following consequence of Theorem 4.2 and Corollary 4.1 .

COROLLARY 4.2. For every $\epsilon>0$ and for every compact subset $K$ of the solution set $T R(c o F)$ of inclusion (8), (9), there exists a continuous map $r_{\epsilon}$ from $K$ to the solution set $T R(F)$ of inclusion (2), (9) such that $\left\|r_{\epsilon}(x)(t, \tau)-x(t, \tau)\right\| \leq \epsilon \forall x \in K,(t, \tau) \in$ $\Pi$.

\section{Controllability and extremality for nonconvex Darboux problems}

We begin this section with the following definition on controllability and extremality in inclusion (2), (9).

DEFINITION 5.1. The inclusion (2), (9) is called $f$-locally controllable around the solution $\bar{x}(t, \tau)$ at point $(T, \mathscr{T})$ if (6) holds. When (6) does not hold then the solution $\bar{x}(t, \tau)$ is called an $f$-extremal solution of inclusion (2), (9).

Obviously every optimal solution of problem (1), (2), (4), (9) is also a $f$-extremal solution of inclusion (2), (9). So necessary conditions for $f$-extremal solutions are also necessary conditions for optimal solutions.

Let us recall some definitions from $[3,27]$.

DEFINITION 5.2. Let $X$ be a Banach space and $K \subset X$. The intermediate tangent cone to $K$ at $a \in \bar{K}$ is the cone

$$
I(K ; a)=\left\{v \in X: \forall h_{i} \rightarrow 0+, \exists v_{i} \rightarrow v \text { such that } a+h_{i} v_{i} \in K\right\} .
$$


DEFINITION 5.3. Let $G: R^{\bar{m}} \rightarrow \operatorname{Comp} R^{n}$ be a set-valued map which is locally Lipschitzian at $a \in \operatorname{Dom} G$ and let $b \in G(a)$. The derivative of $G$ at $(a, b)$ is the set-valued map $d G(a, b): R^{m} \rightarrow 2^{R^{n}}$ such that

$$
v \in d G(a, b)(u) \Leftrightarrow(u, v) \in I(\operatorname{graph} G ;(a, b)),
$$

or equivalently

$$
v \in d G(a, b)(u) \Leftrightarrow \lim _{h \rightarrow 0+} \operatorname{dist}\left(v, \frac{G(a+h u)-b}{h}\right)=0 .
$$

We refer to [3] for general properties of $I(K ; a)$ and $d G(a, b)$.

DEFINITION 5.4. Let $K \subset R^{n}$ and $a \in \bar{K}$. A closed convex cone $\mathscr{M}$ is said to be a regular tangent cone to $K$ at $a$ if there exist continuous functions $q_{h}: \mathscr{M} \cap S_{n} \rightarrow$ $R^{n} \forall h>0$ satisfying

$$
\lim _{h \rightarrow 0+} \max _{v \in \mathscr{M} \cap S_{n}}\left\|q_{h}(v)\right\| / h=0
$$

and $a+h v+q_{h}(v) \in K \forall h>0, v \in \mathscr{M} \cap S_{n}$.

This cone is not uniquely defined and when $K$ is convex then the cone $I(K ; a)$ is also a regular tangent cone to $K$ at $a$ [27].

Now, let $\{A(t, \tau),(t, \tau) \in \Pi\}\left(\left\{A_{1}(t), t \in \Pi_{1}\right\},\left\{A_{2}(\tau), \tau \in \Pi_{2}\right\}\right.$, respectively) be a family of closed convex processes from $R^{n} \times R^{n} \times R^{n}\left(R^{n}, R^{n}\right.$, respectively) to $R^{n}$ satisfying the following assumptions.

C.1. For all $(u, p, q) \in R^{n} \times R^{n} \times R^{n}\left(u \in R^{n}, u \in R^{n}\right.$, respectively) the map $(t, \tau) \rightarrow A(t, \tau)(u, p, q)\left(t \rightarrow A_{1}(t) u, \tau \rightarrow A_{2}(\tau) u\right.$, respectively) is measurable on $\Pi\left(\Pi_{1}, \Pi_{2}\right.$, respectively).

C.2. For all $(t, \tau) \in \Pi\left(t \in \Pi_{1}, \tau \in \Pi_{2}\right.$, respectively) the map $(u, p, q) \rightarrow$ $A(t, \tau)(u, p, q)\left(u \rightarrow A_{1}(t) u, u \rightarrow A_{2}(\tau) u\right.$, respectively) is Lipschitzian with Lipschitz constant $k_{1}>0$.

C.3. graph $A(t, \tau) \subset$ graphdco $\left.F\left(t, \tau, Z(\bar{x})(t, \tau), \bar{x}_{t \tau}(t, \tau)\right)\right)$ for almost all $(t, \tau) \in \Pi$, graph $A_{1}(t) \subset$ graph $d F_{1}\left(t, \bar{x}(t, 0), \bar{x}_{t}(t, 0)\right)$ for amost all $t \in \Pi_{1}$, graph $A_{2}(\tau) \subset$ graph $F_{2}\left(\tau, \bar{x}(0, \tau), \bar{x}_{\tau}(0, \tau)\right)$ for almost all $\tau \in \Pi_{2}$, where $d \operatorname{coF}\left(t, \tau, Z(\bar{x})(t, \tau), \bar{x}_{t \tau}(t, \tau)\right)\left(d F_{1}\left(t, \bar{x}(t, 0), \bar{x}_{t}(t, 0)\right), d F_{2}(\tau, \bar{x}(0\right.$, $\left.\tau), \bar{x}_{\tau}(0, \tau)\right)$, respectively) is the derivative of $\operatorname{co} F(t, \tau, \cdot, \cdot, \cdot)\left(F_{1}(t, \cdot), F_{2}(\tau, \cdot)\right.$, respectively) at $\left(Z(\bar{x})(t, \tau), \bar{x}_{t \tau}(t, \tau)\right) \in \operatorname{graph} \operatorname{coF}(t, \tau, \cdot, \cdot, \cdot)\left(\left(\bar{x}(t, 0), \bar{x}_{t}(t, 0)\right)\right.$ $\in \operatorname{graph} F_{1}(t, \cdot),\left(\bar{x}(0, \tau), \bar{x}_{\tau}(0, \tau)\right) \in \operatorname{graph} F_{2}(\tau, \cdot)$, respectively).

Let $\mathscr{M}$ be a regular tangent cone to $F_{0}$ at $\bar{x}(0,0)$. To the inclusion (2), (9) let us associate the inclusion

$$
\begin{aligned}
w_{t \tau}(t, \tau) & \in A(t, \tau)(Z(w)(t, \tau)),(t, \tau) \in \Pi, \\
w_{t}(t, 0) & \in A_{1}(t) w(t, 0), \quad t \in \Pi_{1}, \\
w_{\tau}(0, \tau) & \in A_{2}(\tau) w(0, \tau), \quad \tau \in \Pi_{2}, \quad w(0,0) \in \mathscr{M} .
\end{aligned}
$$


Let $\mathscr{R}(A)$ denote the solution set of the inclusion (56), (57) and as before $T R(F)$ and $T R(c o F)$ the solution sets of inclusions (2), (9) and (8), (9) respectively. It is obvious that $\mathscr{R}(A)$ is a convex cone in $Q$, and moreover

LEMMA 5.1 [42, Theorem 3.1]. Let there be given functions $w^{i} \in \mathscr{R}(A), i=$ $1,2, \ldots, \ell+1$ and let functions $w(\gamma) \in \mathscr{R}(A)$ be defined by $w(\gamma)=\sum_{i=1}^{\ell+1} \gamma_{i} w^{i}$ for $\gamma \in \mathscr{E}^{\ell}$. Then for every $\delta>0$ one can find a $\epsilon_{0}>0$ such that there exist $x_{\epsilon_{0}}(\cdot, \cdot, \gamma) \in T R($ coF $)$ satisfying

$$
\begin{aligned}
& x_{\epsilon_{0}}(t, \tau, \gamma)=\bar{x}(t, \tau)+\epsilon_{0} w(\gamma)(t, \tau)+o\left(\epsilon_{0}, \gamma, t, \tau\right), \quad(t, \tau) \in \Pi, \\
& \max _{\gamma \in \bar{\Phi}^{\ell}}\left\|o\left(\epsilon_{0}, \gamma, \cdot, \cdot\right)\right\|_{Q} / \epsilon_{0} \leq \delta,
\end{aligned}
$$

and the map $\gamma \rightarrow o\left(\epsilon_{0}, \gamma, \cdot, \cdot\right)$ from $\mathscr{E}^{\ell}$ into $Q$ is continuous.

The following result of Warga on an open mapping theorem plays an important role in deriving our main results.

LEMMA $5.2\left[48\right.$, Theorem 2.3]. Let $\delta \leq 1, g(\cdot)$ be a map from $R^{n}$ to $R^{m}$ which is $C^{1}$ in a neighborhood of zero containing $\delta S_{n}$. Assume that for some $\beta>0$ and every $\theta \in \delta \overline{\mathscr{E}}^{n}$

$$
\beta S_{m} \subset \nabla g(\theta) \overline{\mathscr{E}}^{n} .
$$

Then for every continuous map $\psi: \delta \overline{\mathscr{E}}^{n} \rightarrow R^{m}$ such that

$$
\sup _{\theta \in \delta \delta^{n}}\|g(\theta)-\psi(\theta)\| \leq \delta \beta / 32,
$$

we have

$$
\psi\left(O_{n}\right)+(\delta \beta / 16) S_{m} \subset \psi\left(\delta \overline{\mathscr{E}}^{n}\right) .
$$

Now we can state the first result of this section.

THEOREM 5.1. Inclusion (2), (9) is $f$-locally controllable around $\bar{x}(t, \tau)$ at point $(T, \mathscr{T})$ if for every $\lambda \in \partial_{x} f(\bar{x}(T, \mathscr{T})$ ) inclusion (56), (57) is $\lambda$-locally controllable around the null solution at the point $(T, \mathscr{T})$, that is, if

$$
O_{m} \in \operatorname{int} \lambda\left(R_{A}(T, \mathscr{T})\right) \quad \forall \lambda \in \partial_{x} f(\bar{x}(T, \mathscr{T})),
$$

where $R_{A}(T, \mathscr{T})$ is the reachable set of the inclusions $(56),(57)$ at the point $(T, \mathscr{T})$. PROOF. The proof proceeds in two steps.

Step 1. First we notice that since $\lambda\left(R_{A}(T, \mathscr{T})\right)$ is a convex cone, the relation (58) implies $R^{m}=\lambda\left(R_{A}(T, \mathscr{T})\right) \forall \lambda \in \partial_{x} f(\bar{x}(T, \mathscr{T}))$. Hence we can show that for every $\beta>0$ there exist $n_{1}$ and $u_{j} \in R_{A}(T, \mathscr{T}), j=1,2, \ldots, n_{1}$, such that

$$
\beta S_{m} \subset \lambda\left(u\left(\mathscr{E}^{n_{1}}\right)\right) \quad \forall \lambda \in \partial_{x} f(\bar{x}(T, \mathscr{T})),
$$


where $u\left(\mathscr{E}^{n_{1}}\right)=\left\{u(\gamma)=\sum_{j=1}^{n_{1}} \gamma_{j} u_{j}: \gamma=\left(\gamma_{1}, \gamma_{2}, \ldots, \cdot, \gamma_{n_{1}}\right) \in \mathscr{E}^{n_{1}}\right\}$.

Indeed, let $b_{j} \in R^{m}, j=1,2, \ldots, m+1$ be such that $2 \beta S_{m} \subset b\left(\mathscr{E}^{m+1}\right)$, where $b(\gamma):=\sum_{j=1}^{m+1} \gamma_{j} b_{j}, \gamma=\left(\gamma_{1}, \gamma_{2}, \ldots, \gamma_{m+1}\right) \in \mathscr{E}^{m+1}$. Clearly there exists $\epsilon>0$ such that $\beta S_{m} \subset b^{\prime}\left(\mathscr{E}^{m+1}\right)$, whenever $\left\|b_{j}-b_{j}^{\prime}\right\| \leq \epsilon, j=1,2, \ldots, m+1$ and $b^{\prime}(\gamma):=\sum_{j=1}^{m+1} \gamma_{j} b_{j}^{\prime}$

For every $\lambda \in \partial_{x} f(\bar{x}(T, \mathscr{T}))$ there exist $u_{\lambda}^{j} \in R_{A}(T, \mathscr{T})$ satisfying $\lambda\left(u_{\lambda}^{j}\right)=$ $b_{j}, j=1,2, \ldots, m+1$. Set $\epsilon_{\lambda}=\epsilon / \max _{j=1,2, \ldots, m+1}\left\|u_{\lambda}^{j}\right\|$ and $\mathscr{N}(\lambda)=\left\{\lambda^{\prime} \in \partial_{x} f(\bar{x}(T\right.$, $\left.\mathscr{T})):\left\|\lambda^{\prime}-\lambda\right\|<\epsilon_{\lambda}\right\}$. Obviously $\left\{\mathscr{N}(\lambda), \lambda \in \partial_{x} f(\bar{x}(T, \mathscr{T}))\right\}$ is a covering of $\partial_{x} \eta(z(T))$, and because the latest is a compact set there exists a finite subcovering $\left\{\mathscr{N}\left(\lambda_{i}\right), i=1,2, \ldots, \ell\right\}$.

For every $\lambda \in \partial_{x} f(\bar{x}(T, \mathscr{T}))$, there exists $i$ such that $\lambda \in \mathscr{N}\left(\lambda_{i}\right)$ and so $\| \lambda\left(u_{\lambda_{i}}^{j}\right)-$ $\lambda_{i}\left(u_{\lambda_{i}}^{j}\right)\|\leq\| \lambda-\lambda_{i}\|.\| u_{\lambda_{i}}^{j}\left\|<\epsilon_{\lambda_{i}}\right\| u_{\lambda_{i}}^{j} \| \leq \epsilon$. Hence $\beta S_{m} \subset \lambda\left(u_{\lambda_{i}}^{j}\left(\mathscr{E}^{m+1}\right)\right)$ with $u_{\lambda_{i}}^{j}(\gamma):=$ $\sum_{j=1}^{m+1} \gamma_{j} u_{\lambda_{i}}^{j}$. If we set $n_{1}=\ell(m+1)$ and $u_{j}=u_{\lambda_{1}}^{j}, j=1,2, \ldots, m+1, u_{m+1+j}=$ $u_{\lambda_{2}}^{j}, j=1,2, \ldots, m+1, \ldots, u_{n_{1}}=u_{\lambda_{\ell}}^{m+1}$, the desired relation (59) follows.

Now from the separation theorem, it is easily seen that there exist $\bar{\beta}>0, \bar{\rho}>0$ such that for every $\lambda \in R^{n \times m}$ satisfying $\operatorname{dist}\left(\lambda, \partial_{x} f(\bar{x}(T, \mathscr{T}))\right) \leq \bar{\rho}$ we have

$$
\bar{\beta} S_{m} \subset \lambda\left(u\left(\mathscr{E}^{n_{1}}\right)\right) \text {. }
$$

Let $w_{j}(t, \tau), j=1,2, \ldots, n_{1}$ be solutions of inclusion $(56),(57)$ such that $w_{j}(T, \mathscr{T})$ $=u_{j}, j=1,2, \ldots, n_{1}$.

Set $w(t, \tau, \gamma)=\sum_{j=1}^{n_{1}} \gamma_{j} w_{j}(t, \tau) \quad \forall \gamma \in R^{n_{1}}$. Obviously $w(t, \tau, \gamma)$ is also a solution of inclusion (56), (57) for every $\gamma \in \mathscr{E}^{n_{1}}$.

For every $e>0$ define

$$
d_{e}=\sup \left\{\operatorname{dist}\left(\nabla f(x), \partial_{x} f(\bar{x}(T, \mathscr{T}))\right):\|x-\bar{x}(T, \mathscr{T})\|<e, x \in R^{n}, \nabla f(x) \text { exists }\right\} .
$$

By the upper semicontinuity of Clarke's generalized Jacobian we have $\lim _{e \rightarrow 0+} d_{e}=0$. In view of Lemma 5.1 and the last written relation we can choose $e_{0}, \epsilon_{0}$, so small that $d_{e_{0}}<\bar{\rho}$,

$$
\left\|\epsilon_{0} w(T, \mathscr{T}, \gamma)\right\| \leq e_{0} / 2 \quad \forall \gamma \in \mathscr{E}^{n_{1}}
$$

and there are solutions $\bar{x}(t, \tau, \gamma)$ of inclusion (8), (9) such that the map $\gamma \rightarrow \bar{x}(\cdot, \cdot, \gamma)$ is continuous and satisfies

$$
\|y(T, \mathscr{T}, \gamma)-\bar{x}(T, \mathscr{T}, \gamma)\|<\epsilon_{0} \bar{\beta} / 128 k \quad \forall \gamma \in \mathscr{E}^{n_{1}}
$$


where

$$
y(t, \tau, \gamma):=\bar{x}(t, \tau)+\epsilon_{0} w(t, \tau, \gamma) .
$$

From Corollary 4.2 there exists $x(t, \tau, \gamma), \gamma \in \mathscr{E}^{n_{1}}$ such that the map $\gamma \rightarrow x(\cdot, \cdot \gamma)$ is continuous from $\mathscr{E}^{n_{1}}$ to $T R(F)$ and satisfies

$$
\|x(T, \mathscr{T}, \gamma)-\bar{x}(T, \mathscr{T}, \gamma)\| \leq \epsilon_{0} \bar{\beta} / 128 k \quad \forall \gamma \in \mathscr{E}^{n_{1}} .
$$

Combining this with (62) yields

$$
\|y(T, \mathscr{T}, \gamma)-x(T, \mathscr{T}, \gamma)\| \leq \epsilon_{0} \bar{\beta} / 64 k \quad \forall \gamma \in \mathscr{E}^{n_{1}}
$$

Step 2. Choose a mollifier $\chi(\cdot): R^{n} \rightarrow[0,1]$, that is, a function $\chi(\cdot)$ of the class $C^{\infty}$ with support in $S_{n}$ satisfying $\int_{R^{n}} \chi(x) d x=1$. For every $x \in R^{n}$ define

$$
f_{0}(x)=\int_{R^{n}} f(x-v y) \chi(y) d y,
$$

where

$$
\nu=\min \left\{e_{0} / 2, \epsilon_{0} \bar{\beta} / 64 k\right\} \text {. }
$$

The function $f_{0}$ is of the class $C^{\infty}$ and satisfies

$$
\begin{aligned}
\left\|f(x)-f_{0}(x)\right\| & \leq k_{1} v \\
\nabla f_{0}(x) & =\int_{R^{n}} \nabla f(x-v y) \chi(y) d y .
\end{aligned}
$$

Hence

$$
\nabla f_{0}(x) \in \operatorname{clco}\{\nabla f(z):\|z-x\| \leq v, \nabla f(z) \text { exists }\} .
$$

Define also $\phi(\gamma)=f_{0}\left(\bar{x}(T, \mathscr{T})+\epsilon_{0} w(T, \mathscr{T}, \gamma)\right)$. Then for every $\mu \in \mathscr{E}^{n_{1}}$ one has

$$
\nabla \phi(\gamma) \mu=\nabla f_{0}\left(\bar{x}(T, \mathscr{T})+\epsilon_{0} w(T, \mathscr{T} \gamma)\right) \epsilon_{0} w(T, \mathscr{T}, \mu)
$$

Because for every $\gamma \in \mathscr{E}^{n_{1}}$,

$$
\begin{aligned}
& \nabla f_{0}\left(\bar{x}(T, \mathscr{T})+\epsilon_{0} w(T, \mathscr{T}, \gamma)\right) \\
& \quad \in c l\left\{\nabla f(z):\left\|z-\left(\bar{x}(T, \mathscr{T})+\epsilon_{0} w(T, \mathscr{T}, \gamma)\right)\right\| \leq e_{0} / 2, \nabla f(z) \text { exists }\right\}
\end{aligned}
$$

it follows that

$$
\begin{aligned}
& \operatorname{dist}\left(\nabla f_{0}\left(\bar{x}(T, \mathscr{T})+\epsilon_{0} w(T, \mathscr{T}, \gamma)\right), \partial_{x} f(\bar{x}(T, \mathscr{T}))\right) \\
& \leq \sup \left\{\operatorname{dist}\left(\nabla f(z), \partial_{x} f(\bar{x}(T, \mathscr{T}))\right)\right):\|z-\bar{x}(T, \mathscr{T})\| \\
& \quad \leq\left\|z-\left(\bar{x}(T, \mathscr{T})+\epsilon_{0} w(T, \mathscr{T}, \gamma)\right)\right\|+\left\|\epsilon_{0} w(T, \mathscr{T}, \gamma)\right\| \\
& \left.\quad \leq e_{0}, \quad \nabla f(z) \text { exists }\right\} \\
& \leq d_{e_{0}}<\bar{\rho} .
\end{aligned}
$$


On combining this with (60) we have $\epsilon_{0} \bar{\beta} S_{m} \subset \nabla \phi(\gamma) \mathscr{E}^{n_{1}} \forall \gamma \in \mathscr{E}^{n_{1}}$. Define the function $\psi$ by setting

$$
\psi(\gamma)=f(x(T, \mathscr{T}, \gamma)) \forall \gamma \in \mathscr{E}^{n_{1}} .
$$

This function is continuous as the composition of the continuous functions $f(\cdot)$ and $x(T, \mathscr{T}, \cdot)$. Moreover, by virture of (63), (64), and (66) for every $\gamma \in \mathscr{E}^{n_{1}}$ we have

$$
\begin{aligned}
\|\psi(\gamma)-\phi(\gamma)\|= & \left\|f(x(T, \mathscr{T}, \gamma))-f_{0}(y(T, \mathscr{T}, \gamma))\right\| \\
\leq & \|f(x(T, \mathscr{T}, \gamma))-f(y(T, \mathscr{T}, \gamma))\| \\
& \quad+\left\|f(y(T, \mathscr{T}, \gamma))-f_{0}(y(T, \mathscr{T}, \gamma))\right\| \\
\leq & k\|x(T, \mathscr{T}, \gamma)-y(T, \mathscr{T}, \gamma)\|+k_{1} \nu \leq \epsilon_{0} \bar{\beta} / 64+\epsilon_{0} \bar{\beta} / 64 \\
= & \epsilon_{0} \bar{\beta} / 32 .
\end{aligned}
$$

Therefore by Lemma 5.2,

$$
\begin{aligned}
f\left(x\left(T, \mathscr{T}, O_{n_{1}}\right)\right)+\left(\epsilon_{0} \bar{\beta} / 16\right) S_{m} \subset \psi\left(\mathscr{E}^{n_{1}}\right) \\
\quad=\left\{f(x(T, \mathscr{T}, \gamma)): \gamma \in \mathscr{E}^{n_{1}}\right\} \subset f\left(R_{F}(T, \mathscr{T})\right) .
\end{aligned}
$$

Since by $(63),\left\|f(\bar{x}(T, \mathscr{T}))-f\left(x\left(T, \mathscr{T}, O_{n_{1}}\right)\right)\right\| \leq \epsilon_{0} \bar{\beta} / 64$, from the above relation we also have $f(\bar{x}(T, \mathscr{T})) \in \operatorname{int} f\left(R_{F}(T, \mathscr{T})\right)$, completing the proof of Theorem 5.1.

REMARK 5.1. The analogous result to Theorem 5.1 when $F$ has convex values and $f \equiv I d$ or $f$ is continuously differentiable, was given in $[42,44]$.

Before going on to the main result let us state the following lemma, which is a direct consequence of Theorem 4, Proposition 2 and Lemma 2 in [43].

LEMMA 5.3. For $b \in R_{A}(T, \mathscr{T})^{+}$there exist functions $\tilde{p}^{i} \in Q, i=1,2,3$ and absolutely continuous functions $\tilde{q}^{1}, \tilde{q}^{2}$ on $\Pi_{1}$ and $\Pi_{2}$ respectively satisfying the following inclusions.

$$
\begin{aligned}
\left(\tilde{p}_{t \tau}^{1}(t, \tau), \tilde{p}_{t \tau}^{2}(t, \tau), \tilde{p}_{t \tau}^{3}(t, \tau)\right) & \in A^{*}(t, \tau)\left(\tilde{p}^{1}(t, \tau)-\tilde{p}_{t}^{2}(t, \tau)-\tilde{p}_{\tau}^{3}(t, \tau)\right) \text { a.e. on } \Pi, \\
\dot{\tilde{q}}^{1}(t) & \in A_{1}^{*}(t)\left(-\tilde{q}^{1}(t)-\tilde{p}_{t}^{2}(t, 0)+\tilde{p}^{1}(t, 0)\right) \text { a.e. on } \Pi_{1}, \\
\dot{\tilde{q}}^{2}(\tau) & \in A_{2}^{*}(\tau)\left(-\tilde{q}^{2}(\tau)-\tilde{p}_{\tau}^{3}(0, \tau)+\tilde{p}^{1}(0, \tau)\right) \text { a.e. on } \Pi_{2},(66)
\end{aligned}
$$

with boundary conditions

$$
\begin{aligned}
& \tilde{p}^{1}(T, \tau)=\tilde{p}^{1}(t, \mathscr{T})=b \quad \forall(t, \tau) \in \Pi, \\
& \tilde{p}^{i}(t, \mathscr{T})=\tilde{p}^{i}(T, \tau)=0, \quad i=2,3 \quad \forall(t, \tau) \in \Pi, \\
& \tilde{p}^{1}(0,0)+\tilde{q}^{1}(0) \in-\mathscr{M}^{+}, \\
& \tilde{p}^{1}(0,0)+\tilde{q}^{2}(0) \in-\mathscr{M}^{+} .
\end{aligned}
$$


We are now in a position to prove the main result of this section.

THEOREM 5.2. If $\bar{x}(t, \tau)$ is an $f$-extremal solution then there exist $\theta \in R^{m},\|\theta\|=1$ and functions $\tilde{p}^{i} \in Q, i=1,2,3, \tilde{q}^{1}, \tilde{q}^{2}$ satisfying (66), (67) with $b \in \partial_{x} f(\bar{x}(T, \mathscr{T}))^{*} \theta$ and such that the following maximum condition holds.

$$
\left\langle\tilde{p}(t, \tau), \bar{x}_{t \tau}(t, \tau)\right\rangle=\max \{\langle\tilde{p}(t, \tau), \vartheta\rangle: \vartheta \in F(t, \tau, Z(\bar{x})(t, \tau))\} \text { a.e. on } \Pi,
$$

for $\tilde{p}(t, \tau):=-\tilde{p}^{1}(t, \tau)+\tilde{p}_{t}^{2}(t, \tau)+\tilde{p}_{\tau}^{3}(t, \tau)$.

ProOF. Let $\{\widehat{A}(t, \tau),(t, \tau) \in \Pi\}$ be a family of convex processes defined by

$$
\widehat{A}(t, \tau)(u, p, q)=\overline{A(t, \tau)(u, p, q)+I\left(\operatorname{coF}(t, \tau, Z(\bar{x})(t, \tau)) ; \bar{x}_{t \tau}(t, \tau)\right)} .
$$

By [43, Proposition 3], $\{\widehat{A}(t, \tau),(t, \tau) \in \Pi\}$ possess the analogous conditions to $\{A(t, \tau),(t, \tau) \in \Pi\}$ (see conditions D.1-D.3) and moreover

$$
\begin{gathered}
A(t, \tau)(u, p, q) \subset \widehat{A}(t, \tau)(u, p, q) \forall(u, p, q) \in R^{3 n},(t, \tau) \in \Pi, \\
\widehat{A}^{*}(t, \tau) u= \begin{cases}A^{*}(t, \tau) u & \text { if } u \in\left(F(t, \tau, Z(\bar{x})(t, \tau))-\bar{x}_{t \tau}(t, \tau)\right)^{+} \\
\emptyset & \text { otherwise. }\end{cases}
\end{gathered}
$$

Now consider the inclusion (56), (57) with $A(t, \tau)$ replaced by $\widehat{A}(t, \tau)$ and denote by $R_{\widehat{A}}(T, \mathscr{T})$ its reachable set at the point $(T, \mathscr{T})$. From Theorem 5.1 there exists $\lambda \in \partial_{x} f(\bar{x}(T, \mathscr{T}))$ such that $0 \notin \operatorname{int} \lambda R_{\widehat{A}}(T, \mathscr{T})$ and from the well-known separation theorem (see for example [31, consequence 11.7.3]) this implies the existence of $\theta \in R^{m},\|\theta\|=1$ satisfying $\langle\theta, \lambda v\rangle \geq 0 \forall v \in R_{\widehat{A}}(T, \mathscr{T})$, or, equivalently, $\lambda^{*} \theta \in$ $R_{\widehat{A}}(T, \mathscr{T})^{+}$.

Now from Lemma 5.3 and (70) we obtain (66), (67). The maximum condition (68) follows from (70), (66). The proof of Theorem 5.2 is complete.

On applying the result of Theorem 5.2, we can obtain the maximum condition for problem (1), (3), (4), (5).

Assume that the function $\phi: \Pi \times R^{n} \times R^{n} \times R^{n} \times V \rightarrow R^{n}$, where $V$ is a compact metric space, satisfies the following conditions.

D.1. The function $\phi$ is measurable in $(t, \tau)$, Lipschitzian in $\left(x^{1}, x^{2}, x^{3}\right)$ with Lipschitz constant and continuous in $v$.

D.2. There exists a constant $M$ such that $\left\|\phi\left(t, \tau, x^{1}, x^{2}, x^{3}, v\right)\right\| \leq M \forall t, \tau, x^{1}, x^{2}$, $x^{3}$.

D.3. For a given solution $\bar{x}(t, \tau)$ and its corresponding control $v(t, \tau)$, the function $f(t, \tau, \cdot, \cdot, \cdot, v(t, \tau))$ is differentiable at $Z(\bar{x})(t, \tau)$. 
Under condition E.1, for $F$ defined by

$$
F\left(t, \tau, x^{1}, x^{2}, x^{3}\right)=\phi\left(t, \tau, x^{1}, x^{2}, x^{3}, V\right),
$$

from Filippov-Castaing Theorem [47, Theorem I.7.10, page 153], the solution sets of the inclusion (2), (3) and the system (5) do coincide.

Set

$$
\begin{aligned}
C_{1}(x, y) & =\partial \phi(Z(\bar{x})(x, y), v(x, y)) / \partial x^{1} \\
C_{2}(x, y) & =\partial \phi(Z(\bar{x})(x, y), v(x, y)) / \partial x^{2} \\
C_{3}(x, y) & =\partial \phi(Z(\bar{x})(x, y), v(x, y)) / \partial x^{3} \\
A(x, y) & =\left(C_{1}(x, y), C_{2}(x, y), C_{3}(x, y)\right) .
\end{aligned}
$$

Then $\left\|C_{i}(t, \tau)\right\| \leq k, i=1,2,3$. Hence $A(x, y)$ satisfies conditions D.1-D.3 and inclusion (66) becomes an equation.

Define

$$
\tilde{p}(t, p):=-\tilde{p}^{1}(t, \tau)+\tilde{p}_{t}^{2}(t, \tau)+\tilde{p}_{\tau}^{3}(t, \tau),
$$

where $\tilde{p}^{1}(t, \tau), \tilde{p}^{2}(t, \tau), \tilde{p}^{3}(t, \tau)$ are solutions of (66), (67).

It is easily seen that $\tilde{p}(t, \tau)$ satisfies the integral equation of Volterra type [34]

$$
\begin{aligned}
\tilde{p}(t, \tau)= & -b-\int_{t}^{T} \int_{\tau}^{\mathscr{T}} C_{1}^{*}(\bar{t}, \bar{\tau}) \tilde{p}(\bar{t}, \bar{\tau}) d \bar{\tau} d \bar{t} \\
& -\int_{\tau}^{\mathscr{T}} C_{2}^{*}(t, \bar{\tau}) \tilde{p}(t, \bar{\tau}) d \bar{\tau}-\int_{t}^{T} C_{3}^{*}(\bar{t}, \tau) \tilde{p}(\bar{t}, \tau) d \bar{t} .
\end{aligned}
$$

So as a consequence of Theorem 5.2 we have the following.

COROLlaRY 5.1. If $\bar{x}(t, \tau)$ is an optimal solution of $(1),(3),(4),(5)$, then there exist $\theta \in R^{m},\|\theta\|=1$ and a solution $\tilde{p}(t, \tau)$ of $(73)$ with $b \in \partial_{x} f(\bar{x}(T, \mathscr{T}))^{*} \theta$ such that the following maximum condition holds for a.e. $(t, \tau) \in \Pi$ :

$$
\begin{aligned}
& \langle\tilde{p}(t, \tau), \phi(t, \tau, Z(\bar{x})(t, \tau), v(t, \tau)\rangle \\
& \quad=\max \{\langle\tilde{p}(t, \tau), \phi(t, \tau, Z(\bar{x})(t, \tau)(t, \tau), \vartheta)\rangle: \vartheta \in V\} .
\end{aligned}
$$

Note that an analogous result to Corollary 5.1 has been proved by Suryanaryana in [38] for (1), (3), (5) (without endpoint constrain (4)) under the stronger assumptions that the function $\phi$ is continuous with respect to all variables, for every $(t, \tau)$ the functions $\left(x^{1}, x^{2}, x^{3}\right) \rightarrow \partial f\left(t, \tau, x^{1}, x^{2}, x^{3}\right) / \partial x^{i}, i=1,2,3$ satisfy Lipschitzian condition, and that the objective function $f_{0}$ is either linear or twice contiunuously differentiable.

Actually we can also prove the following result. 
THEOREM 5.3. Suppose that the function $f$ is continuously differentiable at $\bar{x}(T, \mathscr{T})$ and that $\mathscr{M}_{1}$ is some regular tangent cone to $F_{3} \subset C \operatorname{Comp} R^{n}$ at $\bar{x}(T, \mathscr{T}) \in F_{3}$. If $f(\bar{x}(T, \mathscr{T})) \in \partial f\left(R_{F}(T, \mathscr{T}) \cap F_{3}\right)$, then there exist $b^{1} \in-\mathscr{M}_{1}^{+}, b^{2} \in R^{n},\left\|\left(b^{1}, b^{2}\right)\right\|=$ 1, functions $\tilde{p}^{i} \in Q, i=1,2,3$, and absolutely continuous functions $\tilde{q}^{1}, \tilde{q}^{2}$ on $\Pi_{1}$ and $\Pi_{2}$ respectively, satisfying (66)-(68) for $b=b^{1}+\nabla f(\bar{x}(T, \mathscr{T}))^{*} b^{2}$.

Proof. Define

$$
\begin{aligned}
& \widetilde{F}_{0}=F_{0} \times F_{3}, \quad \tilde{u}=\left(u^{1}, u^{2}\right) \in R^{2 n}, \quad \tilde{p}=\left(p^{1}, p^{2}\right) \in R^{2 n}, \quad \tilde{q}=\left(q^{1}, q^{2}\right) \in R^{2 n}, \\
& \mathscr{M}_{0}=\mathscr{M} \times \mathscr{M}_{1}, \\
& \widetilde{F}(t, \tau, \tilde{u}, \tilde{p}, \tilde{q})=\left\{(v, 0), v \in F\left(t, \tau, u^{1}, p^{1}, q^{1}\right)\right\} \quad \forall \tilde{u}, \tilde{p}, \tilde{q},(t, \tau) \in \Pi \\
& \widetilde{F}_{1}(t, \tilde{u})=\left\{(v, 0), v \in F_{1}\left(t, u^{1}\right)\right\} \quad \forall \tilde{u}, t \in \Pi_{1} \\
& \widetilde{F}_{2}(\tau, \tilde{u})=\left\{(v, 0), v \in F_{2}\left(\tau, u^{1}\right)\right\} \quad \forall \tilde{u}, \tau \in \Pi_{2} .
\end{aligned}
$$

It is obvious that the maps $\widetilde{F}\left(\widetilde{F}_{1}, \widetilde{F}_{2}\right.$, respectively) possess analogous conditions to $F\left(F_{1}, F_{2}\right.$, respectively). Consider the inclusion

$$
\begin{aligned}
\tilde{x}_{t \tau}(t, \tau) & \in \widetilde{F}(t, \tau, Z(\tilde{x})(t, \tau)), \quad(t, \tau) \in \Pi, \\
\tilde{x}_{t}(t, 0) & \in \widetilde{F}_{1}(t, \tilde{x}(t, 0)), \quad \tilde{x}_{\tau}(0, \tau) \in \widetilde{F}_{2}(\tau, \tilde{x}(0, \tau)), \\
\tilde{x}(0,0) & \in \mathscr{M}_{0} .
\end{aligned}
$$

Clearly the function $y(t, \tau):=(\bar{x}(t, \tau), \bar{x}(T, \mathscr{T}))$ is a solution of this inclusion.

Define also the families of convex processes $\{\widetilde{A}(t, \tau),(t, \tau) \in \Pi\},\left\{\widetilde{A}_{1}(t), t \in \Pi_{1}\right\}$, $\left\{\widetilde{A_{2}}(\tau), \tau \in \Pi_{2}\right\}$ as follows.

$$
\begin{aligned}
\tilde{A}(t, \tau)(\tilde{u}, \tilde{p}, \tilde{q}) & =\left(\widehat{A}(t, \tau)\left(u^{1}, p^{1}, q^{1}\right), 0\right) \quad \forall(\tilde{u}, \tilde{p}, \tilde{q}), \quad(t, \tau) \in \Pi, \\
\tilde{A}_{1}(t) \tilde{u} & =\left(A_{1}(t) u^{1}, 0\right) \quad \forall \tilde{u}, \quad t \in \Pi_{1}, \\
\tilde{A}_{2}(\tau) \tilde{u} & =\left(A_{2}(\tau) u^{1}, 0\right) \quad \forall \tilde{u}, \quad \tau \in \Pi_{2},
\end{aligned}
$$

where $\widehat{A}(t, \tau)$ is defined by (69).

With the inclusion (76) let us associate the inclusion

$$
\begin{aligned}
\tilde{w}_{t \tau} & \in \widetilde{A}(t, \tau)(Z(\tilde{w})(t, \tau)), \quad(t, \tau) \in \Pi \\
\widetilde{w}_{t}(t, 0) & \in \widetilde{A}_{1}(t) \widetilde{w}(t, 0), \quad t \in \Pi_{1}, \quad \widetilde{w}_{\tau}(0, \tau) \in \tilde{A}_{2}(\tau) \widetilde{w}(0, \tau), \quad \tau \in \Pi_{2}, \\
\widetilde{w}(0,0) & \in \mathscr{M}_{0} .
\end{aligned}
$$

Denote by $\mathscr{R}(\widetilde{F})$ and $\mathscr{R}(\widetilde{A})$ the solution sets of the inclusions (76), (77) respectively and by $R_{\widetilde{F}}(t, \tau), R_{\tilde{A}}(t, \tau)$ their reachable sets at the point $(t, \tau)$.

Define a linear operator $\widetilde{\Lambda}: R^{2 n} \rightarrow R^{n+m}$ by setting

$$
\widetilde{\Lambda}\left(u^{1}, u^{2}\right)=\left(u^{1}-u^{2}, \nabla f(\bar{x}(T, \mathscr{T})) u^{1}\right) .
$$


From Theorem 5.1 we claim that

$$
O_{m} \notin \operatorname{int} \widetilde{\Lambda} R_{\widetilde{A}}(T, \mathscr{T}) .
$$

Indeed, if $O_{m} \in \operatorname{int} \widetilde{\Lambda} R_{\tilde{A}}(T, \mathscr{T})$, then by Theorem $5.1,\left(O_{m}, f(\bar{x}(T, \mathscr{T}))\right) \in \operatorname{int}\left\{\left(x^{1}(T\right.\right.$, $\left.\left.\mathscr{T})-x^{2}(T, \mathscr{T}), f\left(x^{1}(T, \mathscr{T})\right)\right): \tilde{x}(\cdot, \cdot)=\left(x^{1}(\cdot, \cdot), x^{2}(\cdot, \cdot)\right) \in \mathscr{R}(\widetilde{F})\right\}$. Therefore there exists $\sigma>0$ such that $f(\tilde{x}(T, \mathscr{T}))+\sigma S_{m} \subset\left\{f\left(x^{1}(T, \mathscr{T})\right): \tilde{x}(\cdot, \cdot)=\right.$ $\left.\left(x^{1}(\cdot, \cdot), x^{2}(\cdot, \cdot)\right) \in \mathscr{R}(\tilde{F}), x^{1}(T, \mathscr{T})-x^{2}(T, \mathscr{T})=0\right\}=f\left(R_{F}(T, \mathscr{T}) \cap F_{3}\right)$, conflicting with $f(\tilde{x}(T, \mathscr{T})) \in \partial f\left(R_{F}(T, \mathscr{T}) \cap F_{3}\right)$.

Because $\tilde{\Lambda} R_{\tilde{A}}(T, \mathscr{T})$ is a cone in $R^{n+m}$, again using [31, consequence 11.7.3], from (78) there exist $b^{1} \in R^{n}, b^{2} \in R^{m},\left\|\left(b^{1}, b^{2}\right)\right\|=1$ satisfying

$$
\tilde{\Lambda}^{*}\left(b^{1}, b^{2}\right)=\left(b^{1}+\lambda^{*} b^{2},-b^{1}\right) \in R_{\tilde{A}}(T, \mathscr{T})^{+} .
$$

It is easily seen that

$$
\begin{aligned}
\widetilde{A}^{*}(t, \tau)(\tilde{u}, \tilde{p}, \tilde{q}) & =\left\{(v, 0) \in R^{2 n}: v \in \widehat{A}^{*}(t, \tau)\left(u^{1}, p^{1}, q^{1}\right)\right\} \\
\widetilde{A}_{1}^{*}(t) \tilde{u} & =\left\{(v, 0) \in R^{2 n}: v \in A_{1}^{*}(t) u^{1}\right\} \\
\widetilde{A}_{2}^{*}(\tau) \tilde{u} & =\left\{(v, 0) \in R^{2 n}: v \in A_{2}^{*}(t) u^{1}\right\}
\end{aligned}
$$

So (79) yields $b^{1}+\nabla f(\bar{x}(T, \mathscr{T}))^{*} b^{2} \in R_{\widehat{A}}(T, \mathscr{T}),-b^{1} \in \mathscr{M}_{1}^{+}$. Theorem 5.3 follows from Lemma 5.3 and (70).

We close the paper by considering the following time optimal problem for the inclusion (2), (9):

$$
x(\bar{t}, \bar{\tau}) \in F_{3}, \bar{t}^{2}+\bar{\tau}^{2} \rightarrow \text { inf, }
$$

where $F_{3} \in \operatorname{Comp} R^{n}, x(t, \tau)$ is a solution of the inclusion (2), (9).

THEOREM 5.4. If $\bar{x}(t, \tau)$ is an optimal solution and $(T, \mathscr{T})$ the corresponding optimal time of the problem (80), then for every regular tangent cone $\mathscr{M}_{1}$ of $F_{3}$ at $\bar{x}(T, \mathscr{T})$ there exist functions $\tilde{p}^{i} \in Q, i=1,2,3$ and absolutely continuous functions $\tilde{q}^{1}, \tilde{q}^{2}$ on $\Pi_{1}$ and $\Pi_{2}$ respectively satisfying (66), (67) with some $b \in-\mathscr{M}_{1}^{+}$.

PROOF. As in the proof of Theorem 5.3, for $\Lambda \tilde{u}=u^{1}-u^{2} \forall \tilde{u} \in R^{2 n}$, the optimality of $\bar{x}(t, \tau)$ and $T, \mathscr{T}$ implies that

$$
O_{m} \notin \Lambda R_{\widetilde{F}}(t, \tau) \forall(t, \tau) \in \Pi /(T, \mathscr{T}) .
$$

We then see that

$$
O_{m} \notin \text { int } \Lambda R_{\tilde{A}}(T, \mathscr{T}) \text {. }
$$

Indeed, assume the contrary, that $O_{m} \in \operatorname{int} \Lambda R_{\tilde{A}}(T, \mathscr{T})$. Then applying a separation theorem yields the existence of $\epsilon_{0}>0$ such that $O_{m} \in \Lambda R_{\tilde{A}}(T-\epsilon, \mathscr{T}-\epsilon)$ whenever 
$0 \leq \epsilon \leq \epsilon_{0}$. Hence by Theorem 5.1, $O_{m} \in \operatorname{int} \Lambda R_{\widetilde{F}}(T-\epsilon, \mathscr{T}-\epsilon)$ which contradicts (81) and so gives (82).

Because $\Lambda R_{\widetilde{A}}(T, \mathscr{T})$ is a cone in $R^{n}$, from (82) and the separation theorem [31, consequence 11.7.3], there exists a $b \in R^{n},\|b\|=1$ satisfying

$$
\langle b, \bar{v}\rangle \geq 0 \quad \forall \bar{v} \in \Lambda R_{\tilde{A}}(T, \mathscr{T}),
$$

or, equivalently,

$$
\Lambda^{*} b=(b,-b) \in R_{\widehat{A}}(T, \mathscr{T})^{+},
$$

which gives $b \in R_{\widehat{A}}(T, \mathscr{T}),-b \in \mathscr{M}_{1}^{+}$. Theorem 5.3 now follows from Lemma 5.3 and (70). The proof is complete.

REMARK 5.2. When $F_{1}(t, u) \in \operatorname{Con} v R^{n}, F_{2}(t, u) \in \operatorname{Conv} R^{n} \forall u \in R^{n},(t, \tau) \in \Pi$ then we can easily prove (see [43, Remark 1]) that in the statements of Theorems 5.2-5.3, the functions $\bar{x}(t, 0), \bar{x}(0, \tau)$ also satisfy the maximum conditions

$$
\begin{aligned}
\left\langle\bar{x}_{t}(t, 0), q_{1}(t)\right\rangle & =\max \left\{\left\langle v^{1}, q_{1}(t)\right\rangle: v^{1} \in F_{1}(t, \bar{x}(t, 0))\right\} \text { a.e. on } \Pi_{1}, \\
\left\langle\bar{x}_{\tau}(0, \tau), q_{2}(\tau)\right\rangle & =\max \left\{\left\langle v^{2}, q_{2}(\tau)\right\rangle: v^{2} \in F_{2}(\tau, \bar{x}(0, \tau))\right\} \text { a.e. on } \Pi_{2},
\end{aligned}
$$

where $q_{1}(t):=\tilde{q}^{1}(t)+\tilde{p}_{t}^{2}(t, 0)-\tilde{p}^{1}(t, 0), q_{2}(\tau):=\tilde{q}^{2}(\tau)+\tilde{p}_{\tau}^{3}(0, \tau)-\tilde{p}^{1}(0, \tau)$.

\section{Appendix}

We now shall prove Theorem 4.1 by using a method developed in [42] and results of [5] on the existence of continuous selections of set-valued maps.

As before, let $\Gamma$ be a separable Banach space. Recall the following definition.

Definition 6.1. Let $X$ be a Banach space. A set-valued map $\Phi: \Gamma \rightarrow 2^{X}$ is said to be lower semicontinuous (l.s.c.) at $\gamma_{0} \in \Gamma$ if for every open set $\Omega \subset X$ satisfying $\Omega \cap \Phi\left(\gamma_{0}\right) \neq \emptyset$ there exists a neighborhood $U\left(\gamma_{0}\right)$ of $\left(\gamma_{0}\right)$ such that $\Omega \cap \Phi(\gamma) \neq$ $\emptyset \forall \gamma \in U\left(\gamma_{0}\right)$.

A set-valued map $\Phi$ is called lower semicontiuous (l.s.c.) if it is l.s.c. at every $\gamma_{0} \in \Gamma$.

Proposition 6.1 ([5, Propositions 2.1,2.2]). Suppose that a map $F_{*}: \Pi \times \Gamma \rightarrow$ Comp $R^{n}$ is $\mathscr{L} \otimes \mathscr{B}(\Gamma)$-measurable, 1.s.c. with respect to $\gamma \in \Gamma$ and there is a continuous map $\sigma: \Gamma \rightarrow L^{1}(\Pi, R)$ such that $\operatorname{dist}\left(O_{n}, F_{*}(t, \tau, \gamma)\right) \leq \sigma(\gamma)(t, \tau)$ a.e. in $\Pi$. 
Suppose that there are continuous maps $\phi: \Gamma \rightarrow L^{1}(\Pi), \psi: \Gamma \rightarrow L^{1}(\Pi)$ such that for every $\gamma \in \Gamma$ the set

$$
\begin{array}{r}
H(\gamma)=c l\left\{v \in L^{1}(\Pi): v(t, \tau) \in F_{*}(t, \tau, \gamma) \text { a.e. in } \Pi,\right. \\
\|v(t, \tau)-\phi(\gamma)(t, \tau)\|<\psi(\gamma)(t, \tau) \text { a.e. in } \Pi\}
\end{array}
$$

is nonempty.

Then the map $H: \Gamma \rightarrow 2^{L^{\prime}(\Pi)}$ admits a continuous selection.

LEMMA 6.1. The function $\gamma \rightarrow \beta(\cdot, \cdot, \gamma)$ defined from (32) is continuous from $\Gamma$ into $L^{1}(\Pi)$.

PROOF. For every fixed $\gamma_{0} \in \Gamma$ it suffices to show that the function $\gamma \rightarrow \beta(\cdot, \cdot, \gamma)$ is continuous at $\gamma_{0}$.

For every $\gamma \in \Gamma$, one has

$$
\begin{aligned}
\int_{\Pi}\left|\beta\left(t, \tau, \gamma_{0}\right)-\beta(t, \tau, \gamma)\right| d \tau d t \\
\leq \int_{\Pi}\left\|y_{t \tau}\left(t, \tau, \gamma_{0}\right)-y_{t \tau}(t, \tau, \gamma)\right\| d \tau d t \\
\quad+\int_{\Pi} \operatorname{haus}\left(G\left(t, \tau, Z\left(y\left(\gamma_{0}\right)\right)(t, \tau), \gamma_{0}\right), G(t, \tau, Z(y(\gamma))(t, \tau), \gamma)\right) \\
\leq \int_{\Pi}\left(\left\|y_{t \tau}\left(t, \tau, \gamma_{0}\right)-y_{t \tau}(t, \tau, \gamma)\right\|+\left\|Z\left(y\left(\gamma_{0}\right)\right)(t, \tau)-Z(y(\gamma))(t, \tau)\right\|\right) d \tau d t \\
\quad+\int_{\Pi} \operatorname{haus}\left(G\left(t, \tau, Z\left(y\left(\gamma_{0}\right)\right)(t, \tau), \gamma_{0}\right), G\left(t, \tau, Z\left(y\left(\gamma_{0}\right)\right)(t, \tau), \gamma\right) d \tau d t .\right.
\end{aligned}
$$

Because in $Q$ the function $y(\cdot, \cdot, \gamma)$ is continuous at $\gamma_{0}$, for every $\eta / 2>0$ one can find $\epsilon_{1}>0$ such that

$$
\int_{\Pi}\left(\left\|y_{t \tau}\left(t, \tau, \gamma_{0}\right)-y_{t \tau}(t, \tau, \gamma)\right\|+\left\|Z\left(y\left(\gamma_{0}\right)\right)(t, \tau)-Z(y(\gamma))(t, \tau)\right\|\right) d \tau d t \leq \eta / 2
$$

whenever $\left\|\gamma-\gamma_{0}\right\| \leq \epsilon_{1} / 2$. Clearly, for $\beta_{1}(t, \tau, \gamma):=\operatorname{haus}\left(G\left(t, \tau, Z\left(y\left(\gamma_{0}\right)\right)(t, \tau), \gamma_{0}\right)\right.$, $G\left(t, \tau, Z\left(y\left(\gamma_{0}\right)\right)(t, \tau), \gamma\right)$, the function $\beta_{1}(t, \tau, \cdot)$ is continuous in $\gamma$ for a.e. $(t, \tau) \in$ $\Pi$. Since the map $\gamma \rightarrow \delta(\gamma)(\cdot, \cdot)$ is continuous from $\Gamma$ into $L^{1}(\Pi)$ and $\beta_{1}(t, \tau, \gamma) \leq$ $2 k\left\|Z\left(y\left(\gamma_{0}\right)\right)(t, \tau)\right\|+\delta\left(\gamma_{0}\right)(t, \tau)+\delta(\gamma)(t, \tau)$, there exists a function $\bar{\beta}(\cdot, \cdot) \in L^{1}(\Pi, R)$ such that $\beta_{1}(t, \tau, \gamma) \leq \bar{\beta}(t, \tau)$ for a.e. $(t, \tau) \in \Pi$ whenever $\gamma \rightarrow \gamma_{0}$. Hence, by the Lebesgue dominated theorem, in $Q$ for $\beta_{2}:=\int_{n} \beta_{1}(t, \tau, \gamma) d \tau d t$ the function $\gamma \rightarrow \beta_{2}(\gamma)$ is continuous at $\gamma_{0}$. Therefore, there exists $\epsilon_{2}>0$ such that $\beta_{2}(\gamma)=$ $\int_{\Pi} \beta_{1}(t, \tau, \gamma) d \tau d t \leq \eta / 2$ whenever $\left\|\gamma-\gamma_{0}\right\| \leq \epsilon_{2}$. So for every $\eta>0$, there exists $\epsilon=\min \left\{\epsilon_{1}, \epsilon_{2}\right\}$ such that $\int_{\Pi}\left|\beta\left(t, \tau, \gamma_{0}\right)-\beta(t, \tau, \gamma)\right| d \tau d t \leq \eta \forall\left\|\gamma-\gamma_{0}\right\| \leq \epsilon$, proving the continuity of function $\gamma \rightarrow \beta(\cdot, \cdot, \gamma)$ at $\gamma_{0}$ and thereby Lemma 6.1. 
The following lemma can be proved easily.

LEMMA 6.2. Let $G_{0}(t, \tau, \gamma):=G(t, \tau, Z(y(\gamma))(t, \tau), \gamma)$. Then for a.e. $(t, \tau) \in \Pi$ the map $G_{0}(t, \tau, \cdot)$ is lower semicontinuous (l.s.c.).

PROOF. Fix $\gamma_{0} \in \Gamma$. It suffices to show that if a consequence $\gamma_{i} \rightarrow \gamma_{0}$ and $h_{i}(t, \tau):=$ $\operatorname{haus}\left(G\left(t, \tau, Z\left(y\left(\gamma_{0}\right)\right)(t, \tau), \gamma_{0}\right), G\left(t, \tau, Z\left(y\left(\gamma_{i}\right)\right)(t, \tau), \gamma_{i}\right)\right)$, then

$$
\lim _{i \rightarrow \infty} h_{i}(t, \tau)=0 \quad \text { for a.e. }(t, \tau) \in \Pi \text {. }
$$

For this, we note first that for a.e. $(t, \tau) \in \Pi$,

$$
\lim _{i \rightarrow \infty} \operatorname{haus}\left(G\left(t, \tau, Z\left(y\left(\gamma_{0}\right)\right)(t, \tau), \gamma_{0}\right), G\left(t, \tau, Z\left(y\left(\gamma_{0}\right)\right)(t, \tau), \gamma_{i}\right)\right)=0
$$

On the other hand, since the map $\gamma \rightarrow y(\cdot, \gamma)$ is continuous, it is easy to see that

$$
\lim _{i \rightarrow \infty} \int_{\Pi}\left\|Z\left(y\left(\gamma_{0}\right)\right)(t, \tau)-Z\left(y\left(\gamma_{i}\right)\right)(t, \tau)\right\| d \bar{\tau} d \bar{t}=0,
$$

showing $\lim _{i \rightarrow \infty}\left\|Z\left(y\left(\gamma_{0}\right)\right)(t, \tau)-Z\left(y\left(\gamma_{i}\right)\right)(t, \tau)\right\|=0$ for a.e. $(t, \tau) \in \Pi$. Hence (84) follows from the fact that

$$
\begin{aligned}
\lim _{i \rightarrow \infty} h_{i}(t, \tau) \leq & \lim _{i \rightarrow \infty} k\left\|Z\left(y\left(\gamma_{0}\right)\right)(t, \tau)-Z\left(y\left(\gamma_{i}\right)\right)(t, \tau)\right\| \\
& +\lim _{i \rightarrow \infty} \operatorname{haus}\left(G\left(t, \tau, Z\left(y\left(\gamma_{0}\right)\right)(t, \tau), \gamma_{0}\right), G\left(Z\left(y\left(\gamma_{0}\right)\right)(t, \tau), \gamma_{i}\right),\right.
\end{aligned}
$$

completing the proof of Lemma 6.2 .

PROOF OF THEOREM 4.1. We shall construct the function $u$ by using a successive approximation process. By applying Proposition 6.1 at every step of the approximation, the proof is analogous to that of Theorem 2.1 in [42].

Indeed, set

$$
\begin{aligned}
& u_{0}(\gamma)(t, \tau)=y(t, \tau, \gamma), \quad p_{0}(\gamma)(t, \tau)=y_{t}(t, \tau, \gamma), \quad q_{0}(\gamma)(t, \tau)=y_{\tau}(t, \tau, \gamma), \\
& G_{0}(t, \tau, \gamma)=G(t, \tau, Z(y(\gamma))(t, \tau), \gamma), \quad a_{i}=\epsilon /\left(2^{i} T \mathscr{T} \bar{a}\right), i=0,1,2, \ldots,
\end{aligned}
$$

where $\bar{a}$ is defined by (35).

From Lemma 6.2, $G_{0}(t, \tau, \cdot)$ is 1.s.c. for a.e. $(t, \tau) \in \Pi$ and from Lemma 6.1, $\psi_{0}$ is a continuous map from $\Gamma$ into $L^{1}(\Pi)$.

For the map $\gamma \rightarrow G_{0}(\gamma)$ given by

$$
G_{0}(\gamma)=\left\{v \in L^{1}(\Pi): v(t, \tau) \in G_{0}(t, \tau, \gamma) \text { a.e. in } \Pi\right\}, \gamma \in \Gamma,
$$


by Proposition 6.2 there is a continuous selection $v_{0}$ of $G_{0}$ satisfying

$$
\left\|v_{0}(\gamma)(t, \tau)-y_{t \tau}(t, \tau, \gamma)\right\| \leq \psi_{0}(\gamma)(t, \tau)+a_{0} .
$$

Sequences of successive approximations $u_{i}, p_{i}, q_{i}, v_{i}$ are defined by setting, for every $i \geq 1$,

$$
\begin{aligned}
u_{i}(\gamma)(t, \tau) & =\int_{0}^{t} \int_{0}^{\tau} v_{i-1}(\gamma)(\bar{t}, \bar{\tau}) d \bar{\tau} d \bar{t}, \\
p_{i}(\gamma)(t, \tau) & =\int_{0}^{\tau} v_{i-1}(\gamma)(t, \bar{\tau}) d \bar{\tau}, \\
q_{i}(\gamma)(t, \tau, \gamma) & =\int_{0}^{t} v_{i-1}(\gamma)(\bar{t}, \tau) d \bar{t}, \\
G_{i}(t, \tau, \gamma) & =G\left(t, \tau, u_{i}(\gamma)(t, \tau), p_{i}(\gamma)(t, \tau), q_{i}(\gamma)(t, \tau)\right), \\
G_{i}(\gamma) & =\left\{v \in L^{1}(\Pi): v(t, \tau) \in G_{i}(t, \tau, \gamma)\right\}, \\
\psi_{i}(\gamma)(t, \tau) & =\operatorname{dist}\left(v_{i-1}(\gamma)(t, \tau), G_{i}(t, \tau, \gamma)\right),
\end{aligned}
$$

and $v_{i}(\gamma)$ is a continuous selection of $G_{i}(\gamma)$ satisfying

$$
\left\|v_{i}(\gamma)(t, \tau)-v_{i-1}(\gamma)(t, \tau)\right\| \leq \psi_{i}(\gamma)(t, \tau)+a_{i} .
$$

From Lemma 6.2, $G_{i}(t, \tau, \cdot)$ is 1.s.c.. From Lemma 6.1 the map $\gamma \rightarrow \psi_{i}(\gamma)$ is continuous. Hence the existence of $v_{i}(\gamma)$ follows from Proposition 6.2.

Note that

$$
\begin{aligned}
\psi_{i}(\gamma)(t, \tau) \leq & \operatorname{haus}\left(G_{i-1}(t, \tau, \gamma), G_{i}(t, \tau, \gamma)\right) \\
\leq & k\left(\left\|u_{i}(\gamma)(t, \tau)-u_{i-1}(t, \tau)\right\|+\left\|p_{i}(\gamma)(t, \tau)-p_{i-1}(\gamma)(t, \tau)\right\|\right. \\
& \left.\quad+\left\|q_{i}(\gamma)(t, \tau)-q_{i-1}(\gamma)(t, \tau)\right\|\right) .
\end{aligned}
$$

The latter implies

$$
\begin{aligned}
\left\|v_{i}(\gamma)(t, \tau)-v_{i-1}(\gamma)(t, \tau)\right\| \leq k & \left(\left\|u_{i}(\gamma)(t, \tau)-u_{i-1}(\gamma)(t, \tau)\right\|\right. \\
& +\left\|p_{i}(\gamma)(t, \tau)-p_{i-1}(\gamma)(t, \tau)\right\| \\
& \left.+\left\|q_{i}(\gamma)(t, \tau)-q_{i-1}(\gamma)(t, \tau)\right\|\right)+a_{i} .
\end{aligned}
$$

Obviously,

$$
\begin{aligned}
\left\|u_{i+1}(\gamma)(t, \tau)-u_{i}(\gamma)(t, \tau)\right\| & \leq \int_{0}^{t} \int_{0}^{\tau}\left\|v_{i}(\gamma)(\bar{t}, \bar{\tau})-v_{i-1}(\bar{t}, \bar{\tau})\right\| d \bar{\tau} d \bar{t}, \\
\left\|p_{i+1}(\gamma)(t, \tau)-p_{i}(\gamma)(t, \tau)\right\| & \leq \int_{0}^{\tau}\left\|v_{i}(\gamma)(t, \bar{\tau})-u_{i-1}(t, \bar{\tau})\right\| d \bar{\tau} \\
\left\|q_{i+1}(\gamma)(t, \tau)-q_{i}(\gamma)(t, \tau)\right\| & \leq \int_{0}^{t}\left\|v_{i}(\gamma)(\bar{t}, \tau)-v_{i-1}(\bar{t} \tau)\right\| d \bar{t}
\end{aligned}
$$


Set

$$
\begin{aligned}
& z_{i-1}^{1}(\gamma)(t, \tau):=2 \int_{0}^{t} \int_{0}^{\tau}[ k^{i-1} \sum_{j=1}^{2} \frac{g^{i-j}(\bar{t}, \bar{\tau}, t, \tau)}{(i-j) !}\left(\psi_{0}(\gamma)(\bar{t}, \bar{\tau})+a_{0}\right) \\
&\left.+k^{i-2} \sum_{j=1}^{2} \frac{g^{i-j-1}(\bar{t}, \bar{\tau}, t, \tau)}{(i-j-1) !} a_{1}+\cdots+a_{i-1}\right] d \bar{\tau} d \bar{t}, \\
& z_{i-1}^{2}(\gamma)(t, \tau):=z_{i-1}^{1}(\gamma)(t, \tau)+\int_{0}^{\tau}\left[k^{i-1} \frac{(\tau-\bar{\tau})^{i-1}}{(i-1) !}\left(\psi_{0}(\gamma)(t, \bar{\tau})+a_{0}\right)\right. \\
&\left.+k^{i-2} \frac{(\tau-\bar{\tau})^{i-2}}{(i-2) !)} a_{1}+\cdots+a_{i-1}\right] d \bar{\tau}, \\
& z_{i-1}^{3}(\gamma)(t, \tau):=z_{i-1}^{1}(\gamma)(t, \tau)+\int_{0}^{t}\left[k^{i-1} \frac{(t-\bar{t})^{i-1}}{(i-1) !}\left(\psi_{0}(\gamma)(\bar{t}, \tau)+a_{0}\right)\right. \\
&\left.+k^{i-2} \frac{(t-\bar{t})^{i-2}}{(i-2) !} a_{1}+\cdots+a_{i-1}\right] d \bar{t} .
\end{aligned}
$$

Repeating for every $\gamma$ the calculations provided in [42, Theorem 2.1, (2.9)-(3.12)], from (85)-(88), for $g$ defined from (34) and $i \geq 2$, we deduce

$$
\begin{aligned}
\left\|u_{i}(\gamma)(t, \tau)-u_{i-1}(\gamma)(t, \tau)\right\| & \leq z_{i-1}^{1}(\gamma)(t, \tau), \\
\left\|p_{i}(\gamma)(t, \tau)-p_{i-1}(\gamma)(t, \tau)\right\| & \leq z_{i-1}^{2}(\gamma)(t, \tau), \\
\left\|q_{i}(\gamma)(t, \tau)-q_{i-1}(\gamma)(t, \tau)\right\| & \leq z_{i-1}^{3}(\gamma)(t, \tau), \\
\left\|v_{i+1}(\gamma)(t, \tau)-v_{i}(\gamma)(t, \tau)\right\| & \leq k \sum_{j=1}^{3} z_{i-1}^{j}(\gamma)(t, \tau)+a_{i} .
\end{aligned}
$$

Furthermore, the sequence $\left\{u_{i}(\gamma)\right\}_{i=0}^{\infty}$ uniformly converges on $\Pi$ to some function $u(\cdot, \cdot, \gamma)$ the sequences $\left\{p_{i}(\gamma)\right\}_{i=0}^{\infty},\left\{q_{i}(\gamma)\right\}_{i=0}^{\infty}$ converge to some functions $p(\cdot, \cdot, \gamma)$, $q(\cdot, \cdot, \gamma)$ respectively, for almost all $t, \tau$ respectively. Also the sequence $\left\{v_{i}(\gamma)\right\}_{i=0}^{\infty}$ is a Cauchy sequence in $L^{1}(\Pi)$ and hence converges to some function $v(\gamma) \in L^{1}(\Pi)$ almost everywhere on $\Pi$. From Lebesgues' dominated convergence theorem, one has $u(t, \tau, \gamma)=\int_{0}^{t} \int_{0}^{\tau} v(\gamma)(\bar{t}, \bar{\tau}) d \bar{\tau} d \bar{t}, p(t, \tau, \gamma)=\int_{0}^{\tau} v(\gamma)(t, \bar{\tau}) d \bar{\tau}, q(t, \tau, \gamma)=$ $\int_{0}^{t} v(\gamma)(\bar{t}, \tau) d \bar{t}$. Moreover, it is easily seen that for a.e. $(t, \tau) \in \Pi$,

$$
v(\gamma)(t, \tau) \in F(t, \tau, u(t, \tau,), p(\gamma)(t, \tau), q(\gamma)(t, \tau)) .
$$

Therefore $u(t, \tau, \gamma)$ is a solution of inclusion (30), (31). Moreover the map $\gamma \rightarrow$ $u(\cdot, \cdot, \gamma)$ is continuous in $Q$ because every successive approximation $u_{i}$ is continuous in $Q$.

On the other hand,

$$
\int_{0}^{t} \int_{0}^{\tau}\left\|v(\gamma)(\bar{t}, \bar{\tau})-y_{\bar{i} \bar{t}}(\bar{t}, \bar{\tau}, \gamma)\right\| d \bar{\tau} d \bar{t} \leq \sum_{i=1}^{\infty} z_{i}^{1}(t, \tau)
$$




$$
\begin{aligned}
\leq \int_{0}^{t} \int_{0}^{\tau} 2(k+1) \exp (k g(\bar{t}, \bar{\tau}, t, \tau)) \\
\quad \times\left(\psi_{0}(\gamma)(\bar{t}, \bar{\tau})+\sum_{i=0}^{\infty} a_{i}\right) d \bar{\tau} d \bar{t} .
\end{aligned}
$$

Finally the desired estimate (33) follows by noting that for every $(t, \tau) \in \Pi$,

$$
\begin{aligned}
& \int_{0}^{t} \int_{0}^{\tau}\left[2(k+1) \exp \left(k(g(\bar{t}, \bar{\tau}, t, \tau)) \sum_{i=0}^{\infty} a_{i}\right] d \bar{\tau} d \bar{t}\right. \\
& \quad \leq \int_{\Pi}\left[2(k+1) \exp (k g(\bar{t}, \bar{\tau}, t, \tau)) \sum_{i=0}^{\infty} a_{i}\right] d \tau d t \\
& \quad=\epsilon .
\end{aligned}
$$

The proof of Theorem 4.1 is complete.

\section{Acknowledgements}

This paper was writtem while the author was visiting the laboratory of Prof. S. Suzuki, Department of Mechanical Engineering, Sophia University, Tokyo, Japan. The author would like to thank Prof. K. L. Teo and an anonymous referee for their helpful comments which improved the presentation of the paper. He also gratefully acknowledges Prof. Y. Hayakawa of Nagoya University for providing the figures in the paper.

\section{References}

[1] N. V. Ahmed and K. L. Teo, Optimal control of distributed parameter systems, (North-Holland, New York, 1981).

[2] J. P. Aubin and A. Cellina, Differential inclusions, (Springer-Verlag, 1984).

[3] J. P. Aubin and H. Frankowska, Set-valued analysis, (Birkhäuser, Boston, 1990).

[4] A. M. Bagirov, "Relaxed variational problem for systems with distributive parameters", Izvestya Acad. Nauk Azerbaizan SSR, seria fiz. tech. and math. nauk N.3 (1977) 46-51 (in Russian).

[5] A. Bressan and G. Colombo, "Extensions and selections of maps with decomposable values", Studia Math. 90 (1988) 69-85.

[6] L. Cesari, "Optimization with partial differental equations in Dieudone-Rashevsky form and conjugate problems", Archive for Rational Mechanics and Analysis 33 (1969) 339-357.

[7] L. Cesari, Optimization theory and applications problems with ordinary differential equations, (Springer-Verlag, 1983).

[8] F. Clarke, Optimization and nonsmooth analysis, (Wiley Interscience, 1983).

[9] R. M. Colombo, A. Fryszkowski, T. Rzezuchowski and Staicu, "Continuous selection of solutions sets of Lipschitzian differential inclusions", Funkcial. Ekvac. 34 (1991) 321-330.

[10] F. S. De Blasi and J. Myjak, "On the structure of the set of solusions of the Darboux problem of hyperbolic equations", Proc. Edinburgh Math. Soc. 29 (1986) 7-14. 
[11] A. I. Egorov, "Optimal control of processes in certain parameter systems", Automat. Remote Control 25 (1964) 557-566.

[12] A. I. Egorov, "Necessary optimality conditions for systems with distributed parameters", Math. Sbornik 69 (1966) 371-421.

[13] G. Emmanuele and A. Villani, "A linear hyperbolic systems and an optimal control problem", $J$. of Opt. Theory and Applications 44 (1984) 213-229.

[14] H. Frankowska, "The maximum principle for an optimal solution to a differential inclusion with end point constraints", SIAM J. Control and Opt. 25 (1987) 145-157.

[15] H. Frankowska, "Local controllability and infinitesimal generators of semi group of set valued maps", SIAM J. Control and Opt. 25 (1987) 412-431.

[16] H. Frankowska, "Contingent cones to the reachable sets of control systems", SIAM J. Control and Opt. 27 (1989) 170-198.

[17] H. Frankowska, "A priori estimates for operational differential inclusions", J. Differential Equations 84 (1990) 100-128.

[18] H. Frankowska and B. Kaskosz, "Linearization and boundary solutions of nonsmooth control systems", Canadian J. Math. 40 (1988) 589-609.

[19] A. Fryszkowski and T. Rzezuchowski, "Continuous versions of Filippov-Wazewski relaxation Theorem", J. Differential Equations 94 (1991) 254-265.

[20] D. Idczak and S. Walczak, "On the controllability of nonlinear Goursat systems", Optimization 23 (1992) 91-98.

[21] B. Kaskosz and S. Lojasiewisz jr., "A maximum principle for generalized control systems", Nonlinear Analysis 9 (1985) 109-130.

[22] B. Kaskosz, "A maximum principle in relaxed controls", Nonlinear Analysis 14 (1990) 357-367.

[23] M. A. Kazemi-Dehkordi, "A method of successive approximations for optimal control of distributed parameter systems", J. Math. Analysis and Applications 133 (1988) 484-497.

[24] E. H. Mahmudov, "Necessary conditions for optimal control problems described by differential inclusions with distributed parameters", Dokl. Akad. Nauk USSR 303 (1988) 29-33 (in Russian).

[25] S. Marano, "Generalized solutions of partial differential inclusions depending on a parameter", Rend. Acad.Naz.Sci XL Mem.Mat. 107 (1989) 281-295.

[26] V. I. Plotnikov and V. I. Sumin, "Optimization of objects with distributed parameters described by Goursat-Darboux systems", U.S.S.R. Comput. Math. and Math. Phys. 12 (1972) 73-92.

[27] E. S. Polovikin and G. V. Smirnov, "On an optimal time problem for differential inclusions", Differentialnye Uravnenya 22 (1986) 1351-1365 (in Russian).

[28] B. N. Pshenichnyi, Convex analysis and extremal problems, (Nauka, Moskow, 1980) (in Russian).

[29] G. Pulvirenti, "Existence theorems for an optimal control problem relative to a linear hyperbolic partial differential equations", J. Opt. Theory and Applications 7 (1971) 109-117.

[30] G. Pulvirenti and G. Santagati, "On the uniquess of the solution to a minimum problem for a control process with distributed parameters", J. Opt. Theory and Applications 28 (1979) 109-124.

[31] R. T. Rockaffellar, Convex analysis, (Princeton University Press, Princeton, NY, 1970).

[32] V. Staicu, "On a non-convex hyperbolic differential inclusion", Proc. Edinburgh Math. Soc. 35 (1992) 375-382.

[33] V. I. Smirnov, Course of higher mathematics, (Vol.5, Moscow, Fiz. Math., 1960) (in Russian).

[34] M. B. Suryanarayana, "On multidimensional integral equations of Volterra type", Pacific J. Math. 41 (1972) 809-828.

[35] M. B. Suryanarayana, "Existence theorems for optimization problems concerning hyperbolic partial differential equations", J. Opt. Theory and Applications 15 (1975) 361-392.

[36] M. B. Suryanarayana, "Sobolev class and Darboux problem", Pacific J. Mathematics 69 (1977) 535-550. 
[37] M. B. Suryanarayana, "Existence theorems for optimization problems concerning linear hyperbolic partial equations without convexity conditions", J. Opt. Theory and Applications 19 (1976) 47-61.

[38] M. B. Suryanarayana, "Necessary conditions for optimization problems with hyperbolic partial differential equations", SIAM J. Control 11 (1973) 130-147.

[39] K. L. Teo, D. J. Clements, Z. S. Wu and K. G. Choo, "Convergence of a strong variational algorithm for relaxed controls involving a class of hyperbolic systems", J. Opt. Theory and Applications 42 (1984) 467-486.

[40] K. L. Teo, C. J. Goh and K. H. Wong, A unified computational approach to optimal control problems, (Longman Scientific and Technical, 1991).

[41] H. D. Tuan, "Asymptotic construction of solutions to differentiable systems with multi-valued right hand side", Ph.D. thesis, Odessa state univesity, 1990 (in Russian).

[42] H. D. Tuan, "On local controllability of hyperbolic inclusions", J. Math. Systems, Estimation and Control 4 (1994) 319-339.

[43] H. D. Tuan, "Contingent and intermediate tangent cones in hyperbolic differential inclusions and necessary optimality conditions", J. Math. Anal. and Appl. 185 (1994) 86-106.

[44] H. D. Tuan, " $f$-local controllability and extremal solutions in systems under uncertainty governed by inclusions", preprint, Institute of Mathematics, Hanoi, 1992.

[45] H. D. Tuan, "On controllability and extremality in nonconvex differential inclusions", to appear.

[46] A. Villani, "On an optimal control problem for a distributed parameter control process with unbounded coefficients", J. Opt. Theory and Applications 34 (1981) 561-577.

[47] J. Warga, Optimal control of differential and functional equations, (Academic Press, 1972).

[48] J. Warga, "Optimization and controllability without differentiability assumption", SIAM J. Control and Opt. 22 (1983) 837-855.

[49] J. Warga, "Controllability, extremality and abnormality in nonsmooth optimal control", J. Opt. Theory and Applications 41 (1983) 239-260.

[50] J. Warga, "An extension of the Kaskosz maximum principle", Applied Mathematics and Optimization 22 (1990) 61-74.

[51] J. Warga and Q. Zhu, "The equivalence of extremals in different representations of unbounded control problems", to appear.

[52] Z. S. Wu and K. L. Teo, "A convex optimal control problem involving a class of linear hyperbolic systems", J. Opt. Theory and Applications 39 (1983) 541-560.

[53] Z. S. Wu and K. L. Teo, "A conditional gradient method for an optimal control problem involving a class of nonlinear second order hyperbolic partial differential equations", J. Math. Analysis and Applications 91 (1983) 376-393.

[54] Z. S. Wu and K. L. Teo, "First order strong variation algorithm for optimal control problem involving hyperbolic systems", J. Opt. Theory and Applications 42 (1984) 561-587.

[55] K. Yosida, Functional analysis, (Sixth edition), (Springer-Verlag, 1980).

[56] Q. Zhu, "Necessary optimality conditions for nonconvex differential inclusion with endpoint constraints", submitted. 